\title{
Exploring the Conformational Transition Between the Fully Folded and Locally Unfolded Substates of the Escherichia coli thiol peroxidase
}

Diego S. Vazquez ${ }^{1,2,}$, Ari Zeida ${ }^{3}$, William A. Agudelo ${ }^{4}$, Mónica Montes ${ }^{5}$, Gerardo Ferrer-Sueta $^{6}$ and Javier Santos ${ }^{7,8, *}$

${ }^{1}$ Laboratorio de Expresión y Plegado de Proteínas, Departamento de Ciencia y Tecnología, Universidad Nacional de Quilmes, Roque Sáenz Peña 352, Bernal, Buenos Aires, Argentina. ${ }^{2}$ Instituto Multidisciplinario de Biología Celular (IMBICE), Calle 526 y Camino General Belgrano, La Plata, Buenos Aires, Argentina. ${ }^{3}$ Departamento de Bioquímica y Centro de Investigaciones Biomédicas (CEINBIO), Facultad de Medicina, Universidad de la República, Avda. General Flores 2125, Montevideo, Uruguay. ${ }^{4}$ Fundación Instituto de Inmunología de Colombia (FIDIC), Avda. 50 N²6-20, Bogotá D.C., Colombia. ${ }^{5}$ Instituto de Química y Fisicoquímica Biológicas (IQUIFIB) "Prof. Dr. Alejandro C. Paladini”, Universidad de Buenos Aires and CONICET, Junín 956, Ciudad Autónoma de Buenos Aires, Argentina. ${ }^{6}$ Laboratorio de Fisicoquímica Biológica, Instituto de Química Biológica and CEINBIO, Facultad de Ciencias, Universidad de la República, Iguá 4225, Montevideo, Uruguay. ${ }^{7}$ Laboratorio de Genómica e Ingeniería de Sistemas Biológicos. Instituto de Biociencias, Biotecnología y Biología Traslacional $\left(\mathrm{iB}^{3}\right)$. Departamento de Fisiología y Biología Molecular y Celular, Facultad de Ciencias Exactas y Naturales, Universidad de Buenos Aires, Intendente Güiraldes 2160, Ciudad Autónoma de Buenos Aires, Argentina. ${ }^{8}$ Consejo Nacional de Investigaciones Científicas y Técnicas, Av. Rivadavia 1917 (C1033AAJ), Ciudad Autónoma de Buenos Aires, Argentina. 


\section{Correspondence}

*Dr. Diego S. Vazquez, Departamento de Ciencia y Tecnología, Universidad Nacional de Quilmes, Roque Sáenz Peña 352 (B1876BXD), Bernal, Buenos Aires, Argentina and Instituto Multidisciplinario de Biología Celular, Calle 526 y Camino General Belgrano (B1906APO), La Plata, Buenos Aires, Argentina.

E-mail: dsvazquez86@gmail.com. TEL:+54 11 4365-7100 ext. 5642. URL: http://www.unq.edu.ar

*Dr. Javier Santos, Departamento de Fisiología y Biología Molecular y Celular, Facultad de Ciencias Exactas y Naturales, Universidad de Buenos Aires, Intendente Güiraldes 2160 (C1428EGA), Ciudad Autónoma de Buenos Aires, Argentina.

E-mail: javiersantosw@gmail.com. TEL: +54 11 5285-7400 ext. 58431. URL: http://www.fbmc.fcen.uba.ar

Running title: Conformational transition of a 2-Cys Prx

Abbreviations: $\mathrm{aMD}$, accelerated molecular dynamics; cMD, conventional molecular dynamics; $\mathrm{C}_{\mathrm{p}}$, peroxidatic cysteine; $\mathrm{C}_{\mathrm{R}}$, resolving cysteine; EcTPx, thiol peroxidase from E. coli; $\mathrm{HB}$, hydrogen bond; Prx, peroxiredoxin; TPx, thiol peroxidase; TRX, thioredoxin.

Keywords: peroxiredoxin family; conformational landscape; protein dynamics; native ensemble; molecular dynamics simulation, accelerated molecular dynamics.

Conflict of interest: The authors declare no conflict of interest. 


\section{Abstract}

Thiol peroxidase from Escherichia coli $(E c \mathrm{TPx})$ is a peroxiredoxin that catalyzes the reduction of different hydroperoxides. During the catalytic cycle of $E c T P x$, the peroxidatic cysteine $\left(\mathrm{C}_{\mathrm{P}}\right)$ is oxidized to a sulfenic acid by peroxide, then the resolving cysteine $\left(\mathrm{C}_{\mathrm{R}}\right)$ condenses with the sulfenic acid of $\mathrm{C}_{\mathrm{P}}$ to form a disulfide bond, which is finally reduced by thioredoxin. Purified EcTPx as dithiol and disulfide behaves as a monomer in close to physiological conditions. Although secondary structure rearrangements are present when comparing different redox states of the enzyme, no significant differences in unfolding free energies are observed under reducing and oxidizing conditions. A conformational change denominated fully folded (FF) to locally unfolded (LU) transition, involving a partial unfolding of $\alpha \mathrm{H} 2$ and $\alpha \mathrm{H} 3$ helices, must occur to enable the formation of the disulfide bond since the catalytic cysteines are $12 \AA$ apart in the FF conformation of EcTPx. To explore this crucial process, the mechanism of the $\mathrm{FF} \rightarrow \mathrm{LU}$ and the $\mathrm{LU} \rightarrow \mathrm{FF}$ transitions were studied using long time scale conventional molecular dynamic simulations and an enhanced conformational sampling technique for different oxidation and protonation states of $\mathrm{C}_{\mathrm{P}}$ and/or $\mathrm{C}_{\mathrm{R}}$. Our results suggest that the $\mathrm{FF} \rightarrow \mathrm{LU}$ transition has a higher associated energy barrier than the refolding $\mathrm{LU} \rightarrow \mathrm{FF}$ process in agreement with the relatively slow experimental turnover number of EcTPx. Furthermore, in silico designed single-point mutants of the $\alpha \mathrm{H} 3$ enhanced locally unfolding events, suggesting that the native FF interactions in the active site are not evolutionary optimized to fully speed-up the conformational transition of wild-type EcTPx. 


\section{Introduction}

Escherichia coli thiol peroxidase (EcTPx, UniProt entry P0A862) is a small and globular protein of 167 residues belonging to the peroxiredoxin (Prx) family [1-4]. EcTPx shares the prototypical thioredoxin fold, characterized by a seven strand $\beta$ sheet core surrounded by five helices with an N-terminal $\beta$ hairpin extension (Figure 1A). Members of the TPx subfamily, originally named p20 or scavengases $[5,6]$, were identified as bacterial atypical 2-Cys Prxs, as both catalytic cysteines (Cys) are located in the same polypeptide molecule, and conform one of the less phylogenetically diverse subfamilies known [7].

EcTPx reduces $\mathrm{H}_{2} \mathrm{O}_{2}$ [8] and alkyl hydroperoxides [9] via a reactive and absolutely conserved peroxidatic Cys $\left(\mathrm{C}_{\mathrm{P}}\right)$ surrounded by the highly conserved PxxxT/SxxC $\mathrm{P}_{\mathrm{P}}$ motif [10] and an Arg residue that constitute the active site [11]. The first step of the catalytic mechanism involves the nucleophilic attack of the $C_{P}$ sulfur atom on the peroxide yielding the sulfenic acid intermediate (Cys-SOH) and a water molecule if the substrate is $\mathrm{H}_{2} \mathrm{O}_{2}$. The progress in the catalytic cycle depends on the presence and location of a second Cys residue (resolving Cys or $C_{R}$, that react with the sulfenic acid yielding a disulfide bond $\left(R-C_{P}-S-S-C_{R}-R\right)$, releasing a water molecule as a leaving group. Finally, the disulfide bond is reduced by thioredoxin (TRX), through a typical thiol-disulfide exchange reaction (Figure 1B, [12]). 


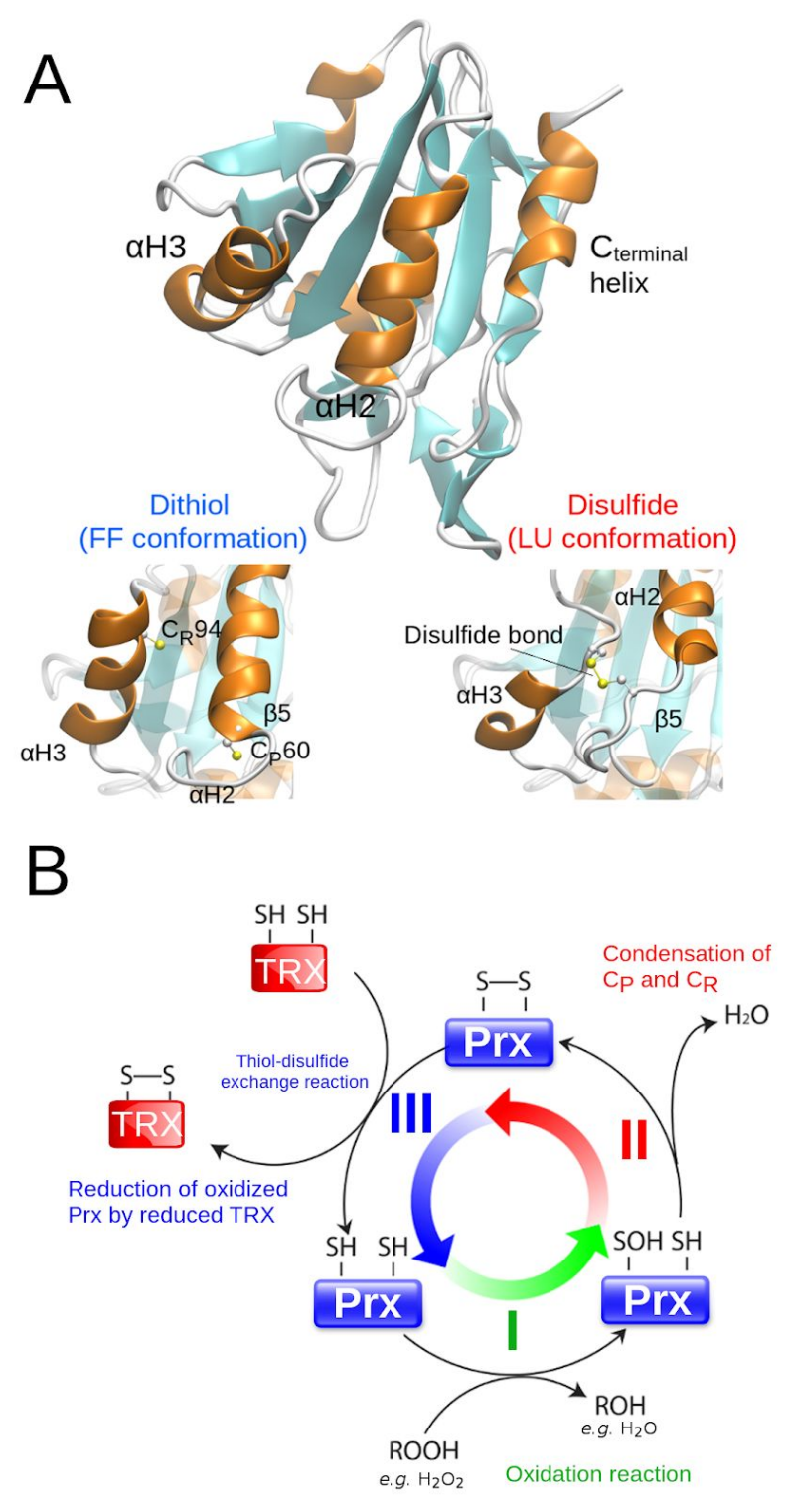

Figure 1: EcTPx X-ray Structure and Catalytic Mechanism. Cartoon representation of the EcTPx structure constituted by a laminar seven strand $\beta$ sheet core surrounded by five helices (Panel A). The FF (reduced state, PDB ID 3HVV) and LU conformations (oxidized state, PDB ID 3HVS chain A) are shown below. The $C_{P}$ and $C_{R}$ are highlighted. The general three-step catalytic cycle for atypical 2-Cys Prxs is shown in panel B and involves: I) the peroxidation of $\mathrm{C}_{\mathrm{P}}$ to form the sulfenic acid intermediate $\left(\mathrm{C}_{\mathrm{P}}-\mathrm{SOH}\right)$; II) the formation of the intramolecular 
disulfide bond between $\mathrm{C}_{\mathrm{P}}$ and $\mathrm{C}_{\mathrm{R}}$, and; III) the reduction of the disulfide by a thiol oxidoreductase (TRX).

The catalytic cycle goes through at least one major conformational transition, the so-called fully folded (FF) to locally unfolded (LU) transition [2,13-15], as $\mathrm{C}_{\mathrm{P}}(\mathrm{Cys} 60)$ and $\mathrm{C}_{\mathrm{R}}$ (Cys94) are more than $12 \AA$ apart in the crystallographic structure of the reduced (dithiol) state (Figure 1A, [13]). Whether the conformational equilibrium is modulated by the redox and protonation state of $\mathrm{C}_{\mathrm{P}}$ and/or $\mathrm{C}_{\mathrm{R}}$ is still an open question. The transition involves a partial unfolding of the C-terminal region of $\alpha \mathrm{H} 3$ (residues 86 to 97), together with a smaller local unfolding of the N-terminal region of $\alpha \mathrm{H} 2$ and the preceding loop connected to the $\beta 5$ strand and changes in the solvent accessibility of $\mathrm{C}_{\mathrm{P}}$ (Figure $\left.1 \mathrm{~A}\right)$.

The specificity constant of $E c \mathrm{TPx}$ for $\mathrm{H}_{2} \mathrm{O}_{2}\left(k_{\text {cat }} / \mathrm{K}_{\mathrm{M}}=8.9 \times 10^{4} \mathrm{M}^{-1} \mathrm{~s}^{-1},[16]\right)$ is in the range observed for other TPx-Prx subfamily members [17]. The kinetic parameters for the individual steps in the reduction of $\mathrm{H}_{2} \mathrm{O}_{2}$ by TPx (catalyzed by TRX) have been reported [8], $k_{\text {cat }}$ $=76 \mathrm{~s}^{-1}\left(\mathrm{H}_{2} \mathrm{O}_{2}\right), \mathrm{K}_{\mathrm{M}}=1.7 \mathrm{mM}\left(\mathrm{H}_{2} \mathrm{O}_{2}\right)$ and $\mathrm{K}_{\mathrm{M}}=25.5 \mu \mathrm{M}(\mathrm{TRX})$, and indicate that the most likely rate-limiting step of the cycle is the peroxidation (Reaction I, Figure 1) since large concentrations of $\mathrm{H}_{2} \mathrm{O}_{2}$ would be needed to saturate the enzyme. The only other hydroperoxide tested, cumene hydroperoxide, has a much smaller $\mathrm{K}_{\mathrm{M}}(9.1 \mu \mathrm{M})$ pointing to a specialization of the enzyme for larger hydrophobic peroxides. In those cases, reduction and/or resolution reactions may become rate-limiting and both reactions involve the conformational transitions under study, $\mathrm{FF} \rightarrow \mathrm{LU}$ and $\mathrm{LU} \rightarrow \mathrm{FF}$, respectively. Crystallographic and kinetic approaches have provided evidence describing the FF and LU conformations in different Prx subfamilies $[8,13,18,19]$. However, a thorough dynamic characterization of the $\mathrm{FF} \rightarrow \mathrm{LU}$ and $\mathrm{LU} \rightarrow \mathrm{FF}$ 
transitions is not available yet. To shed light on this process, the mechanism of EcTPx conformational transitions with $\mathrm{C}_{\mathrm{P}}$ modeled in the thiol, thiolate, sulfenic acid and sulfenate states and $\mathrm{C}_{\mathrm{R}}$ modeled in the thiol and thiolate states were studied in the present work, using long time scale conventional molecular dynamic (cMD) simulations to study the intrinsic dynamics of the FF and LU conformational substates together with the enhanced sampling technique accelerated molecular dynamics (aMD) to describe conformational changes. Additionally, a set of biophysical experiments were carried out in order to characterize the oligomeric state, the secondary/tertiary structure content and the thermodynamic properties of the dithiol and disulfide states of the enzyme.

\section{Results}

\section{Expression, Purification and Experimental Characterization of Wild-type EcTPx}

Escherichia coli TPx was overexpressed as a soluble protein and purified in the oxidized form (disulfide bond between $C_{P} 60$ and $C_{R} 94$ ), as judged by the absence of free thiols in the native state (data not shown). The molecular weight (MW) was evaluated by ES-MS (17701 Da) and coincides within $1 \mathrm{Da}$ with the expected mass deduced from protein sequence, considering the loss of two protons in the oxidized state and the lack of the initial Met residue. The secondary and tertiary structure content of EcTPx in the dithiol (preincubated with $1 \mathrm{mM}$ DTT) and disulfide states were evaluated by circular dichroism (CD) spectroscopy. The spectrum in the far-UV region show native-like signatures compatible with a folded protein (Figure 2A). The lower negative signal at $222 \mathrm{~nm}$ and lower positive signal at $195 \mathrm{~nm}$ of the far-UV CD spectrum in the oxidized form account for the loss of periodic helical structure compatible with the locally 
unfolded (LU) conformation. Furthermore, the near-UV CD spectrum shows a significant gain of negative band in the $270-285 \mathrm{~nm}$ region, in oxidizing conditions, which is also compatible with the formation of a disulfide bond (Figure 2B), reflecting a change in the asymmetric environment of aromatic residues near the active site (Figure 1B).
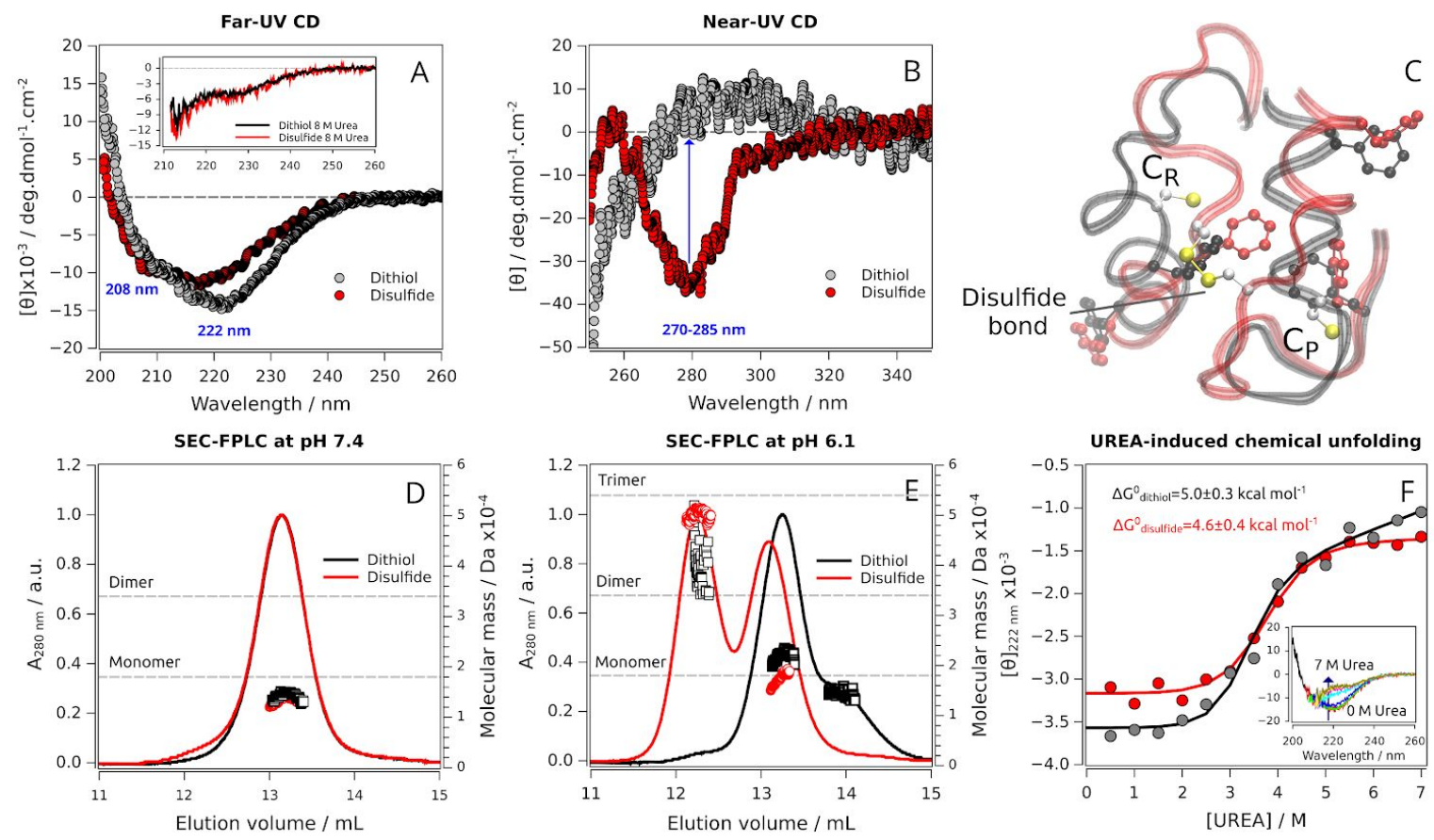

Figure 2: Biophysical Characterization of Wild-type EcTPx. The far-UV (A) and near-UV CD spectra (B) are shown for the reduced (black) and oxidized (red) states. Additionally, the spectra for each oxidation state in the presence of $8.0 \mathrm{M} \mathrm{GdmCl}$ are shown in the inset. Aromatic residues near the active site in the dithiol (black) and disulfide (red) states area highlighted in panel C. The SEC-FPLC elution profile and the oligomeric state was studied by static light scattering at pH 7.4 (D) and pH 6.1 (E) in reduced (black line) and oxidized (red line) conditions, respectively. The theoretical mass of the monomer $(17.7 \mathrm{kDa})$, homodimer $(35.4 \mathrm{kDa})$ and homotrimer $(53.1 \mathrm{kDa})$ are represented by dashed grey lines. The urea-induced chemical 
unfolding of the dithiol and disulfide states of wild-type EcTPx is shown in panel F. Far-UV CD spectra of reduced EcTPX at different urea concentrations are shown in the inset.

A previous bioinformatic and structural analysis reported that the TPx subfamily shows a region of highly conserved hydrophobic residues that in the oxidized state forms an intermolecular interface involving a surface area of $\sim 1400 \AA^{2}[8]$, suggesting a role for the stabilization of the dimeric form of EcTPx in solution. In the same fashion, experimental measurements carried out by analytical ultracentrifugation showed that the dimer is the predominant species in solution above $40 \mu \mathrm{M}$. Furthermore, the authors concluded that the dimer interface is involved in the stabilization of the active site [8]. To evaluate the oligomeric state of EcTPx at low micromolar concentrations $(10-20 \mu \mathrm{M})$, we performed SEC-FPLC experiments monitored by means of a multiangle light scattering detector at neutral $\mathrm{pH}$ and moderate ionic strength (20 mM Tris- $\mathrm{HCl}, 100 \mathrm{mM} \mathrm{NaCl}, \mathrm{pH}$ 7.4). The predominant species in both reduced and oxidized conditions exhibited molecular masses compatible with a monomer species (Figure 2D). These observations are also in agreement with SEC experiments carried out with a TPX from Helicobacter pylori [20] and the PrxQ from Xanthomonas campestris [21] that shows a predominant monomeric species in both reduced and oxidized conditions. To test the effect of ionic strength in the monomer-dimer equilibrium, SEC-FPLC experiments were carried out increasing the $\mathrm{NaCl}$ concentration up to $400 \mathrm{mM}$. No dimeric species were observed, neither in reduced nor oxidized conditions (data not shown).

Curiously, the oxidized enzyme at $\mathrm{pH} 6.1$ forms a homotrimer with an average molecular mass of $49.9 \pm 1.1 \mathrm{kDa}$ (Figure $2 \mathrm{E}$ ). On the other hand, only a much less abundant and highly-dispersed population, compatible with dimeric or trimeric forms, was detected for the 
reduced state. Even more, a species with a molecular mass compatible with the monomer was observed with a slightly higher elution volume, compared to that observed at $\mathrm{pH} 7.4$ (Figure 2D and E), possibly due to a particular interaction of the protein with the Superose-12 matrix at this $\mathrm{pH}$.

The urea-induced chemical unfolding of the reduced and oxidized states of EcTPx are cooperative, indicating that both states are organized and contain a native protein core. The difference in free energy between the unfolded and folded state obtained by equilibrium unfolding experiments are nearly unchanged in the oxidized or reduced states of EcTPx (Figure $2 \mathrm{~F}, \Delta \mathrm{G}_{\mathrm{NU}}^{\circ}<0.5 \mathrm{kcal} \mathrm{mol}^{-1}$ ) which is in agreement with the low difference in the melting temperature $\left(T_{\mathrm{m}}\right)$ observed for the TPx from H. pylori [20] in the reduced $\left(T_{\mathrm{m}}=66.6{ }^{\circ} \mathrm{C}\right)$ and oxidized conditions $\left(T_{\mathrm{m}}=65.0^{\circ} \mathrm{C}\right)$. However, it is worthy to note that the unfolded state of the dithiol and disulfide states of EcTPx is structurally different because of the presence of the disulfide bond, making difficult the comparison between them in energetics terms.

\section{Structural Characterization of the Native FF and LU Conformational Substates by MD}

\section{Simulations}

To get insights about the conformational transitions and internal motions that take place during the catalytic cycle of EcTPx, we performed molecular dynamics (MD) simulations. Several systems were evaluated: (I) the wild-type EcTPx substituting Ser60 residue for Cys $\left(\mathrm{C}_{\mathrm{P}}\right)$ as thiol, thiolate, sulfenic acid or sulfenate (starting from FF conformation, PDB ID 3HVV); (II) the wild-type $E c$ TPx with $\mathrm{C}_{\mathrm{P}}$ as thiol, thiolate, sulfenic acid or sulfenate starting from a locally-unfolded-like conformation (LU*, PDB ID 3HVS chain A); (III) the wild-type EcTPx in the oxidized state (disulfide bond, PDB ID 3HVS chain A); (IV) as system II but evaluating the 
four possible protonation combinations for both $\mathrm{Cys}\left(\mathrm{C}_{\mathrm{P}}\right.$ and $\left.\mathrm{C}_{\mathrm{R}}\right) ;(\mathrm{V})$ three single-point mutants of the aH3 helix, starting from FF conformation, with $\mathrm{C}_{\mathrm{P}}$ and $\mathrm{C}_{\mathrm{R}}$ modeled in the sulfenic acid and thiol form, respectively, substituting the residues Arg92, Phe93 or Glu97 of $\alpha \mathrm{H} 3$ helix by Gly (see text below).

In order to validate our computational simulation setup, a comparative analysis was performed between the root-mean-square deviation (RMSD) of the experimental structures of Bacillus subtilis TPx (BsTPx) determined by NMR [19] in the truly reduced state (dithiol) with the B factor of the crystallographic structures of EcTPx [8] and the root-mean-square fluctuation (RMSF) of our conventional MD (cMD) simulations in the dithiol and disulfide states (Figure 3). Remarkably, our cMD simulations of EcTPx captures the fundamental conformational features of the reduced and oxidized ensembles. The RMSF profiles found in our simulations reproduce very well the ensemble heterogeneities inferred from $B$ factors and RMSD profiles corresponding to EcTpx and BsTPx, respectively. 

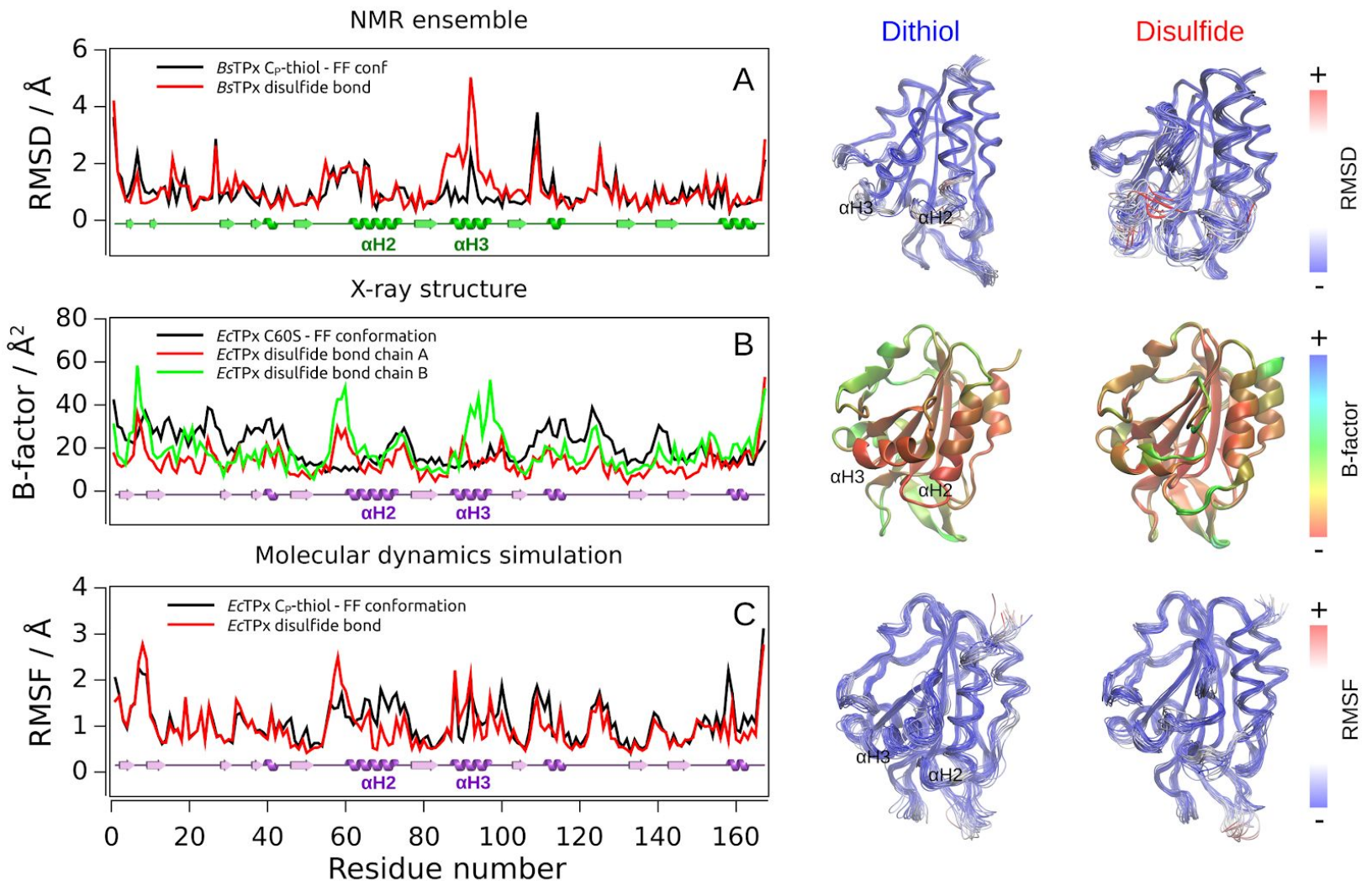

Figure 3: Conformational Heterogeneity of E. coli and B. subtilis TPx. The root-mean-square deviation per residue (RMSD) of the 20 lowest-energy structures of BsTPx determined by NMR in the reduced state (PDB ID 2JSZ, black line) and the disulfide bond state (PDB ID 2JSY, red line) are shown in panel A. The B-factor of EcTPx in the reduced state (PDB ID 3HVV, black line) and disulfide bond state (PDB ID 3HVS chains A and B, red and green lines, respectively) are shown in panel B. The root-mean-square fluctuations (RMSF) of the cMD simulations of both redox states of EcTPx are shown in panel $\mathrm{C}$ with $\mathrm{C}_{\mathrm{P}}$ and $\mathrm{C}_{\mathrm{R}}$ modeled as thiols. The values are represented in the corresponding 3D structure in the right panel. The linear secondary structure and the $\alpha \mathrm{H} 2$ and $\alpha \mathrm{H} 3$ regions are indicated for clarity.

These analyses pointed out the possibility to explore the dynamics of the FF and LU ensembles for the physiological and relevant chemical states of $\mathrm{C}_{\mathrm{P}}$ : thiol, thiolate, sulfenic acid and sulfenate species. In this regard, there is a clear difference in the interactions that each 
species can form and how they may influence in the FF $\leftrightarrows$ LU transition. The thiolate species is a hydrogen bond (HB) acceptor and can establish salt bridge interactions due to its negative net charge, so it is expected to stabilize the FF conformation through interactions with polar and charged residues in the vicinity, particularly the conserved Thr57 and Arg132 of the active site. On the other hand, the thiol group is a poor HB donor due to the low electronegativity and large size of the sulfur atom, moreover, it cannot establish ionic interactions. Once the $\mathrm{C}_{\mathrm{P}}$ is oxidized, the presence of the $\mathrm{C}_{\mathrm{P}}-\mathrm{SOH}$ group, that can form stronger $\mathrm{HB}$ due to the extra oxygen atom, could affect the HB network of the active site. Additionally, being more voluminous than thiol or thiolate, the sulfenic acid species may affect the conformational architecture of the active site [22]. Furthermore, if the sulfenate is the predominant species at neutral $\mathrm{pH}$ [23], the oxygen can act also as an HB acceptor.

We clusterized different structures obtained in the cMDs using principal component analysis (PCA) as clustering criteria and identify key residues involved in large conformational movements. For reduced and oxidized ensembles, the first two eigenvectors fall near the origin of the Cartesian axis, suggesting that the systems are sampling conformations close to the ones observed in the crystallographic structures. Moreover, the overlap of the root-mean-square inner product (RMSIP) value of 0.4 , denotes a low similarity of the overall dynamics [24]. It is worthy to note that the essential subspace in each conformational substate is expected to change due to the conformational restriction imparted by the disulfide bond between $C_{P}$ and $C_{R}$.

The deprotonated $\mathrm{C}_{\mathrm{P}}$-thiolate and $\mathrm{C}_{\mathrm{P}}$-sulfenate species keep nearly unchanged with respect to the starting structure, establishing ionic interactions with Thr57 and Arg132. Although the protonated forms transiently got closer between the catalytic Cys residues, in any case, local 
unfolding transitions were observed, as judged by the secondary structure analysis (SSC, data not shown). This observation suggests that the thiolate species, that is the most abundant form under physiological conditions and reactive one, contributes to the stability of FF conformations.

For the disulfide bond formation, two things must occur: the approximation of catalytic Cys residues and also the loss of secondary structure, with a concomitant increment in local fluctuation. When cMD simulations started from the LU conformation, the deprotonated species, particularly the $\mathrm{C}_{\mathrm{P}}$-thiolate system, present a maximum peak with a $\mathrm{C}_{\mathrm{P}}-\mathrm{C}_{\mathrm{R}}$ distance of $5.6 \pm 0.3$ $\AA$, close to the starting distance at the beginning of the simulation (Figure 4, right panel). Nevertheless, fully refolding events compatible with the FF conformation was not observed in any case. These results are interesting because, although the form that reacts with $C_{R}$ is the sulfenic acid form of $C_{P}$, the sulfenate species would remain closer to the $C_{R}$, transiently stabilizing the LU conformation, necessary for the reaction. Additionally, it has been found that although the preferent reaction is between $C_{P}$ sulfenic and $C_{R}$ thiolate, the reaction with the $C_{P}$ sulfenate also occurs [23]. 

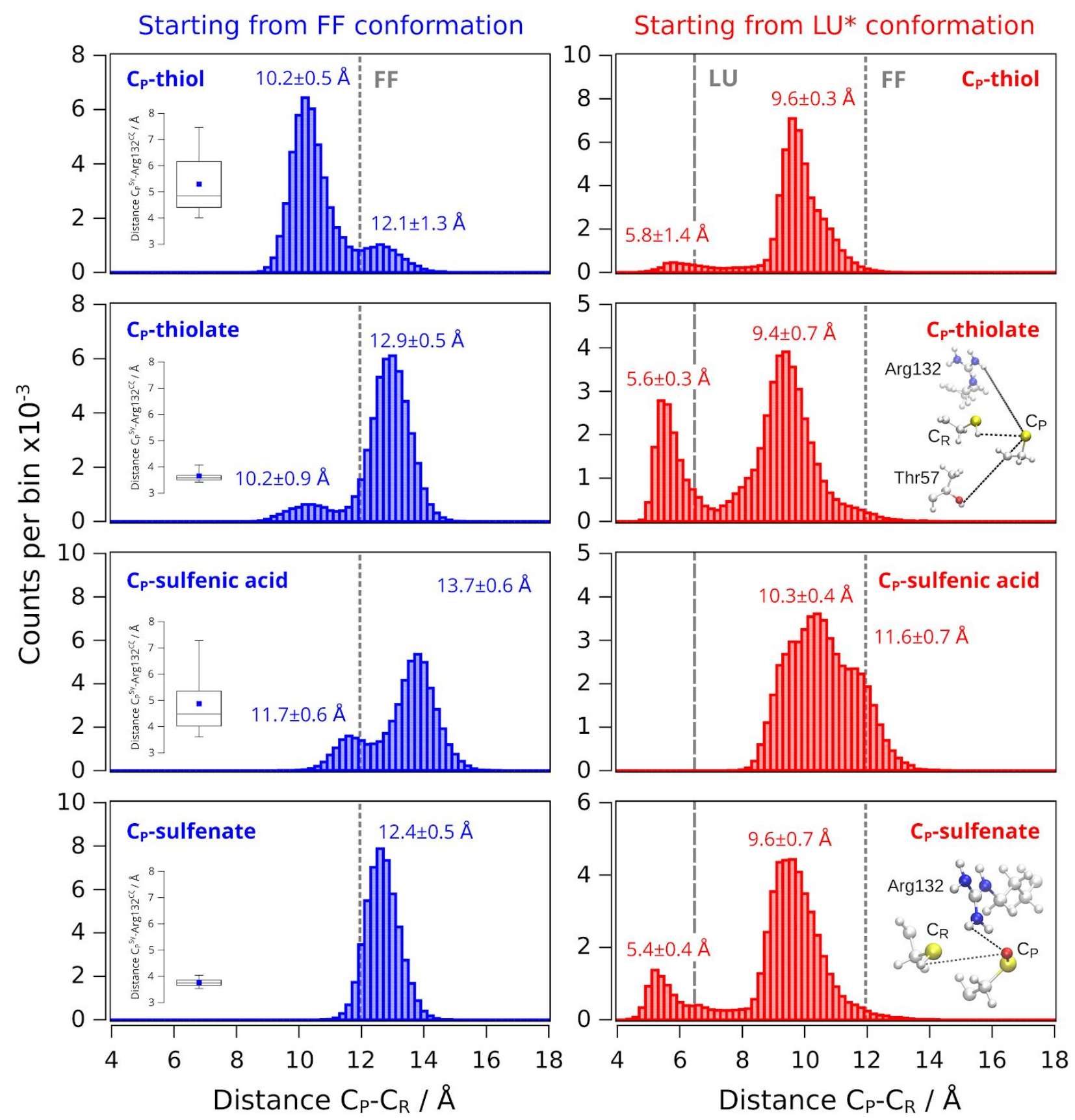

Figure 4: Analysis of $C_{P}-C_{R}$ Distance Distributions. Histogram The $\mathrm{C}_{\mathrm{P}}-\mathrm{C}_{\mathrm{R}}$ distance of the $\mathrm{C} \alpha$ atoms through the cMD simulations starting from the FF (left panel) or LU* (right panel) conformations for different oxidation and protonation states of $\mathrm{C}_{\mathrm{P}}$. The distributions of the distance between the sulfur atom of $\mathrm{C}_{\mathrm{P}}$ and the $\mathrm{C} \zeta$ atom of $\operatorname{Arg} 132$ are shown as boxplot insets. The $\mathrm{C}_{\mathrm{R}}$ was modeled as a thiol in all systems. Vertical grey lines indicate the distance values 
between $\mathrm{C} \alpha$ atom in the corresponding crystallographic conformation. The values reported corresponds to the mean $\pm 1 \sigma$ of the multimodal Gaussian fit.

\section{Exploring the $\mathrm{FF} \rightarrow \mathrm{LU}$ Conformational Transition by Accelerated MD Simulations}

Helix-coil transitions usually involve surpassing high energy barriers, thus cMD simulations become insufficient to study this kind of conformational rearrangements. To overcome this obstacle, we employed accelerated molecular dynamics (aMD) simulations, an enhanced sampling technique that extends the effective simulation time scale to the order of microseconds [25]. Due to the unknown value of the energy barrier involved in the FF $\rightarrow$ LU process, four multiples $\lambda$ values of the acceleration factor $\alpha$ were applied to the EcTPx $\mathrm{C}_{\mathrm{P}}$-sulfenic acid in the $\mathrm{FF}$ conformation system ranging from 0.3 to 4.0 collecting a total $1.75 \times 10^{9}$ simulation steps, equivalent to a computational cost of $3.5 \mu \mathrm{s}$. Although at $\lambda=0.3$ the system shows a more spread out map along the first two eigenvectors compared with the cMD simulation (Figure 5A), the conformations sampled in the cluster of maximal frequency are still similar to those sampled by the cMD simulation, as pointed out by the low value of the average $\triangle$ RMSD of $0.5 \AA$, a RMSIP value of 0.7 and a $C_{P}-C_{R}$ distance of $12.2 \pm 0.8 \AA$ between $C \alpha$ atoms. Interestingly, multiple solvent exposure events of the $\mathrm{C}_{\mathrm{P}}$ residue were observed at $\lambda=1.0$ characterized by a $\triangle \mathrm{SASA}$ of $88.2 \pm 20.2 \AA^{2}$. Nevertheless, this was not accompanied by a significant local unfolding of the $\alpha \mathrm{H} 2$ neither an approach of the catalytic Cys which keep at an average distance of $12.1 \pm 0.7 \AA$ (Figure 5B). This fact could indicate that the Cys approximation and the concomitant condensation of the disulfide bond, involves two independent stages where the unfolding of one of the helices does not induce the unfolding of the other. Similar results 
were observed at $\lambda=2.0$ where the $C_{P}-C_{R}$ distance remained stable at $11.9 \pm 0.6 \AA$ without evidencing significant changes in the SSC of $\alpha \mathrm{H} 2$ or $\alpha \mathrm{H} 3$ (Figure 5C).

It is worth to note that an increase in $\lambda$ parameter (i.e. a decrease in the acceleration factor $\alpha$ ) will result in a decrease in $\Delta \mathrm{V}(r)$ (the boost potential) and then, a longer simulation time will be needed to properly sample the protein conformational space [26]. For this reason, a microsecond time scale simulation was performed at $\lambda=4.0$. The bidimensional heatmap shows more populated minima besides the crystallographic basin (Figure 5D). To characterize the conformations accumulated in each energy minimum, a clustering analysis over the projection of the covariance matrix in the accelerated simulation was conducted. Five minima clusters preserving native and non-native signatures were found at $\lambda=4.0$ and four of them are characterized to hold conformations which are far away from the crystallographic FF conformational substate. The conformations clustered in the well 1 have a slight shift in the position of $\alpha \mathrm{H} 2$ and a twist of $40^{\circ}$ in the $\alpha \mathrm{H} 3$ refereed to the cMD simulation with no significant loss of helicity. For wells 2 to 5, a drastic sheet-to-helix transition involved the antiparallel $\beta 3-\beta 4$ (residues 22 to 44 ) to an $\alpha$-helix was formed. The loss of the $\beta 3-\beta 4$ interactions affects the local dynamics of $\beta 7$ which is in close proximity with the $\alpha \mathrm{H} 3$ helix. Nevertheless, a similar behaviour was observed for $\alpha \mathrm{H} 2$ and $\alpha \mathrm{H} 3$ than in the well 1 . Well 3 holds structures in which the helix observed in the wells 2-5 breaks into 2 parallel $\alpha$-helix with a partial unfolding of $\alpha \mathrm{H} 3$ and interestingly near $70 \%$ of the conformations have the catalytic Cys at less than $4 \AA$. Surprisingly, a complete loss of secondary structure of the $\alpha \mathrm{H} 3$ helix was found in well 4 but the average $\mathrm{C}_{\mathrm{P}}-\mathrm{C}_{\mathrm{R}}$ distance remained at $12.1 \pm 0.8 \AA$ and only $5 \%$ of the conformers the catalytic 
Cys approach to less than $4 \AA$, reinforcing the idea about the independence between $C_{P}-C_{R}$ approximation and local unfolding phenomena.
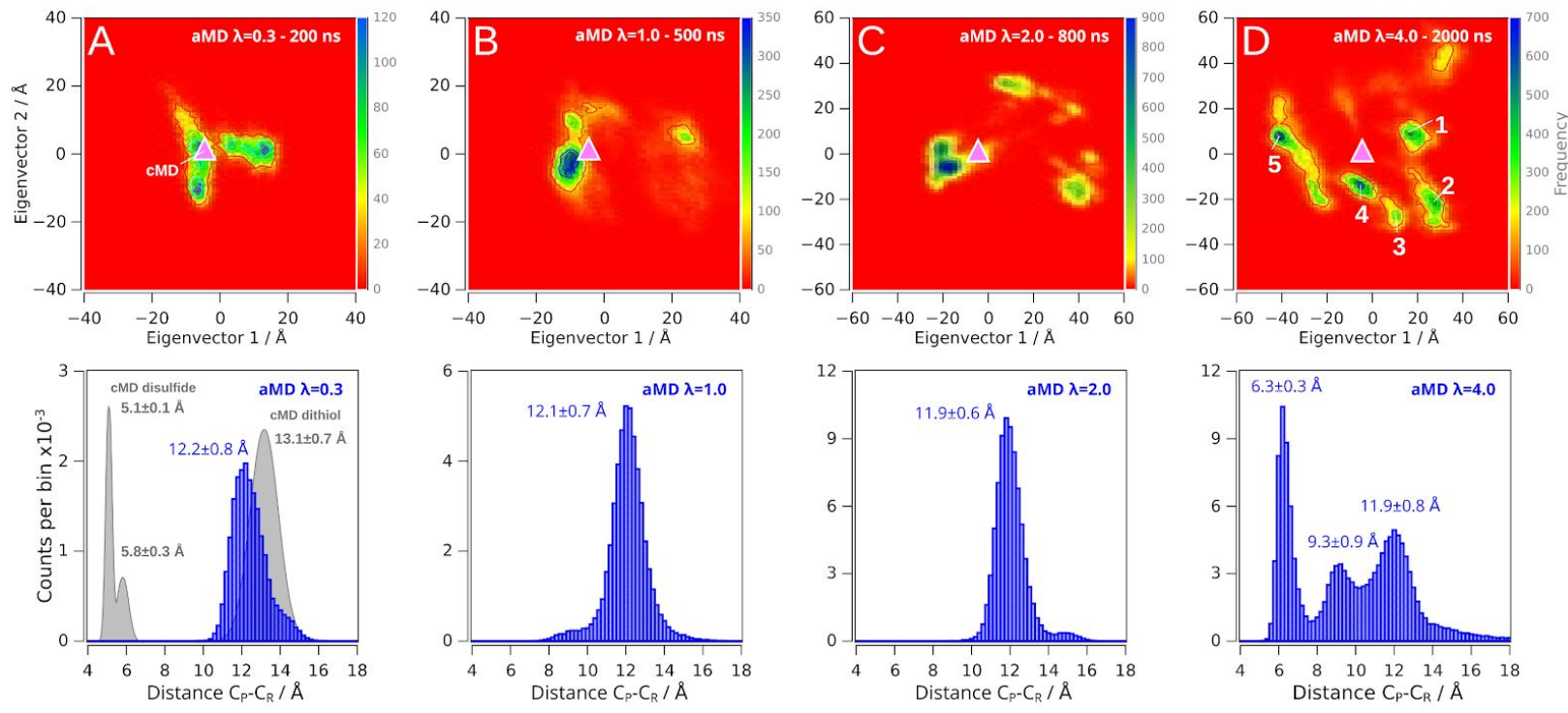

Figure 5: Effect of $\lambda$ Factor on the Conformational Sampling of Wild-type EcTPx. Bidimensional heatmaps of the eigenvectors 1 and 2 projected onto the aMD trajectory for $\lambda$ factor values of 0.3 (A), 1.0 (B), 2.0 (C), and 4.0 (D) are shown in the top. The pink triangle denotes the position of the maximum frequency in the cMD simulation starting from the FF conformational substate with $C_{P}$ and $C_{R}$ modeled as the sulfenic acid and thiol, respectively. The numbers in panel D denotes the wells analyzed in the main text. The corresponding histogram of the $C_{P}-C_{R}$ distance for $\mathrm{C} \alpha$ atoms through all the trajectory is shown below each panel. The distributions for the dithiol and disulfide states of the cMD simulations are shown as a reference (grey). The values reported corresponds to the mean $\pm 1 \sigma$ of the multimodal Gaussian fit.

\section{Exploring the $\mathrm{LU} \rightarrow$ FF Conformational Transition by Accelerated MD Simulations}

To explore the conformational transition from the LU to the FF conformation, we prepared four additional systems using a LU-like (LU*) conformation as starting structures where the $C_{P}$ and $C_{R}$ (in the reduced state) were combined in the protonated $(P)$ or deprotonated 
(D) states (I: $\mathrm{C}_{\mathrm{P}}{ }^{\mathrm{P}}$ and $\mathrm{C}_{\mathrm{R}}{ }^{\mathrm{P}}$; II: $\mathrm{C}_{\mathrm{P}}{ }^{\mathrm{P}}$ and $\mathrm{C}_{\mathrm{R}}{ }^{\mathrm{D}}$; III: $\mathrm{C}_{\mathrm{P}}{ }^{\mathrm{D}}$ and $\mathrm{C}_{\mathrm{R}}{ }^{\mathrm{P}}$; and $\mathrm{IV}$ : $\mathrm{C}_{\mathrm{P}}{ }^{\mathrm{D}}$ and $\mathrm{C}_{\mathrm{R}}{ }^{\mathrm{D}}$ ). The systems were subjected to $100 \mathrm{~ns}$ of cMD simulation using a distance restraint of $3.5 \AA$ between the $\mathrm{C} \alpha$ atoms of $\mathrm{C}_{\mathrm{P}}$ and $\mathrm{C}_{\mathrm{R}}$. Then four aMD simulation replicas $\left(7.5 \times 10^{7}\right.$ simulation steps per replica, equivalent to $150 \mathrm{~ns}$ ) were performed for each system using the last structure as initial coordinates for the unrestricted simulations assigning new random velocities.

The analysis of the simulation of the $\mathrm{C}_{\mathrm{P}}{ }^{\mathrm{P}}-\mathrm{C}_{\mathrm{R}}{ }^{\mathrm{P}}$ system showed an ensemble population with a local RMSD of $2.2 \AA$ and $\mathrm{a}_{\mathrm{P}}-\mathrm{C}_{\mathrm{R}}$ distance distribution of $12.4 \pm 0.8 \AA$ between $\mathrm{C} \alpha$ atoms (Figure 6). The analysis of the helical content per residue (Figure 7A) through the simulations, revealed that the protonation of $\mathrm{C}_{\mathrm{R}}$ rises the helical propensity of the first segment of $\alpha \mathrm{H} 3$ comprising the residues 87 to 90 , to more than $90 \%$ on the whole trajectory. Furthermore, when the simulations were analyzed in three sections: the first part (0-50 ns), seconds part (50-100 ns), and the third part (100-150 ns) we observed that during the last part of the simulations (100-150 ns) the helical structure of the complete $\alpha \mathrm{H} 3$ (residues 87-96) was acquired (Figure 7A). On the other hand, when both Cys residues were modeled as thiolate (system IV, $\mathrm{C}_{\mathrm{P}}{ }^{\mathrm{D}}$ and $\mathrm{C}_{\mathrm{R}}{ }^{\mathrm{D}}$ ), the stabilization of helical structure for the first seven residues of $\alpha \mathrm{H} 3$ (residues 87-93) was significantly reduced. Intermediate behaviors were observed for systems II and III which have one Cys as thiolate and the other as thiol $\left(\mathrm{C}_{\mathrm{P}}{ }^{\mathrm{P}}-\mathrm{C}_{\mathrm{R}}{ }^{\mathrm{D}}\right.$ and $\mathrm{C}_{\mathrm{P}}{ }^{\mathrm{D}}-\mathrm{C}_{\mathrm{R}}{ }^{\mathrm{P}}$, Figures 6 and 7).

Deprotonated Cys residues established ionic pair interactions with Arg92, Arg65 (in the case of $C_{P}$ ) and $\operatorname{Arg} 132$ (in the case of $C_{R}$, Figure 7B). These interactions presumably impede the helical structure acquisition, or at least delay its induction, stabilizing the LU conformations and keeping the system trapped, suggesting that the protonation state of the $C_{P}$ residue is a key feature for the modulation of the equilibrium between the LU and FF conformations. Noticeably, 
Arg65, Arg92 and Arg132 residues are conserved in the TPx subfamilies [1]. This behavior is also in agreement with a high local RMSD of the $\alpha \mathrm{H} 3$ helix and broad distribution of the $\mathrm{C}_{\mathrm{P}}-\mathrm{C}_{\mathrm{R}}$ distance (Figure 6).
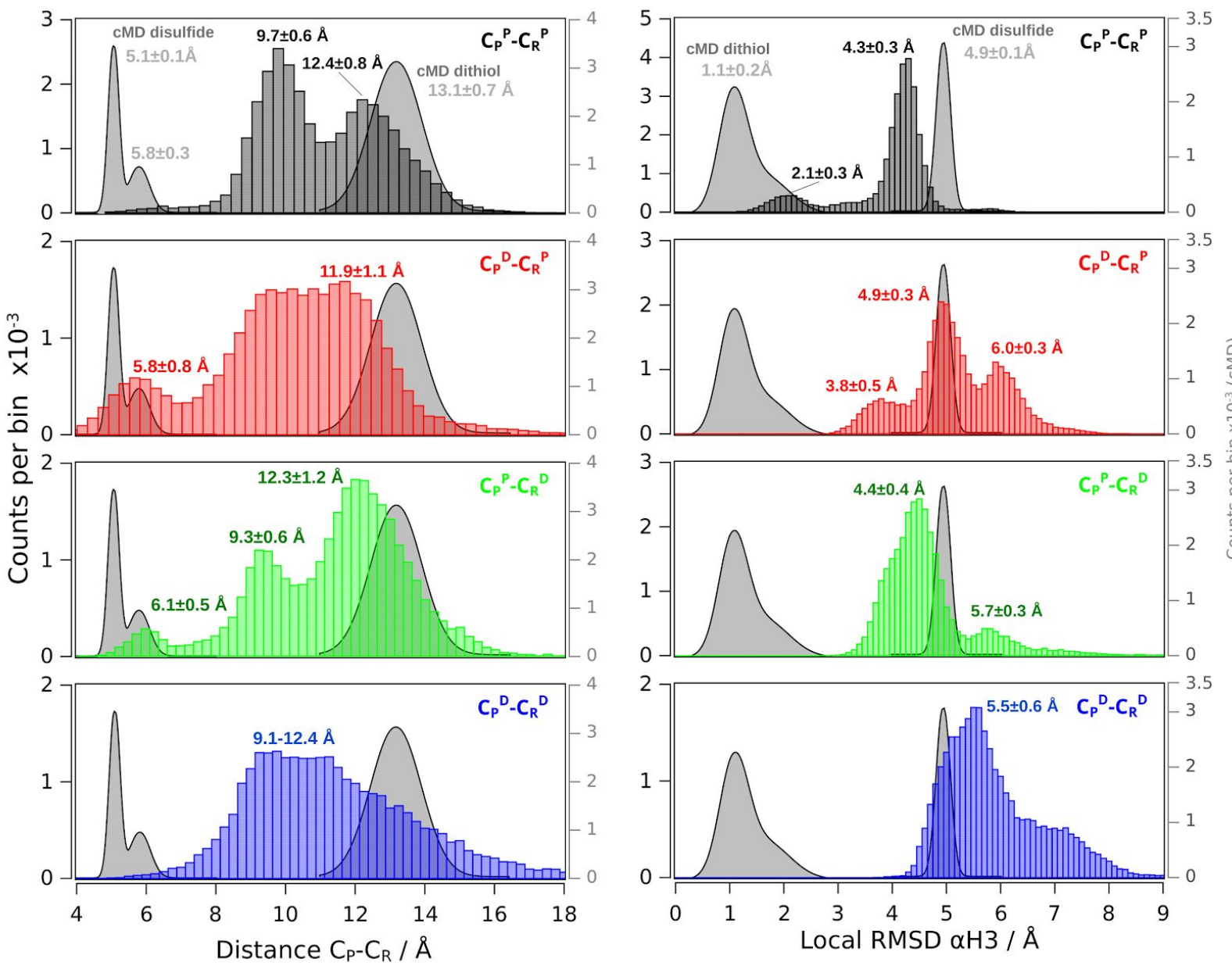

Figure 6: Effect of Protonation State of $C_{P}$ and $C_{R}$ on the Acquisition of the $L U \rightarrow F F$ Conformational Transition. Distribution of local RMSD of the $\alpha \mathrm{H} 3$ was performed over the 150 ns aMD simulation for the $\mathrm{C}_{\mathrm{P}}{ }^{\mathrm{P}}-\mathrm{C}_{\mathrm{R}}{ }^{\mathrm{P}}$ (black), $\mathrm{C}_{\mathrm{P}}{ }^{\mathrm{P}}-\mathrm{C}_{\mathrm{R}}{ }^{\mathrm{D}}$ (red), $\mathrm{C}_{\mathrm{P}}{ }^{\mathrm{D}}-\mathrm{C}_{\mathrm{R}}{ }^{\mathrm{P}}$ (green) and $\mathrm{C}_{\mathrm{P}}{ }^{\mathrm{D}}-\mathrm{C}_{\mathrm{R}}{ }^{\mathrm{D}}$ (blue) systems are shown in the right panel. The crystallographic FF conformation (PDB ID 3HVV) was used for all systems as reference for global fitting and subsequent local RMSD calculation of the $\alpha \mathrm{H} 3$. The histograms of the $\mathrm{C}_{\mathrm{P}}$ and $\mathrm{C}_{\mathrm{R}}$ distance between the $\mathrm{C} \alpha$ atoms are shown in the left panel. The distance distribution for the FF and LU systems in the cMD simulations is shown as a reference (grey). The values reported corresponds to the mean $\pm 1 \sigma$ of the multimodal Gaussian 
fit. The superscript $\mathrm{P}$ and $\mathrm{D}$ labels denote the protonated or deprotonated state of the catalytic Cys, respectively.
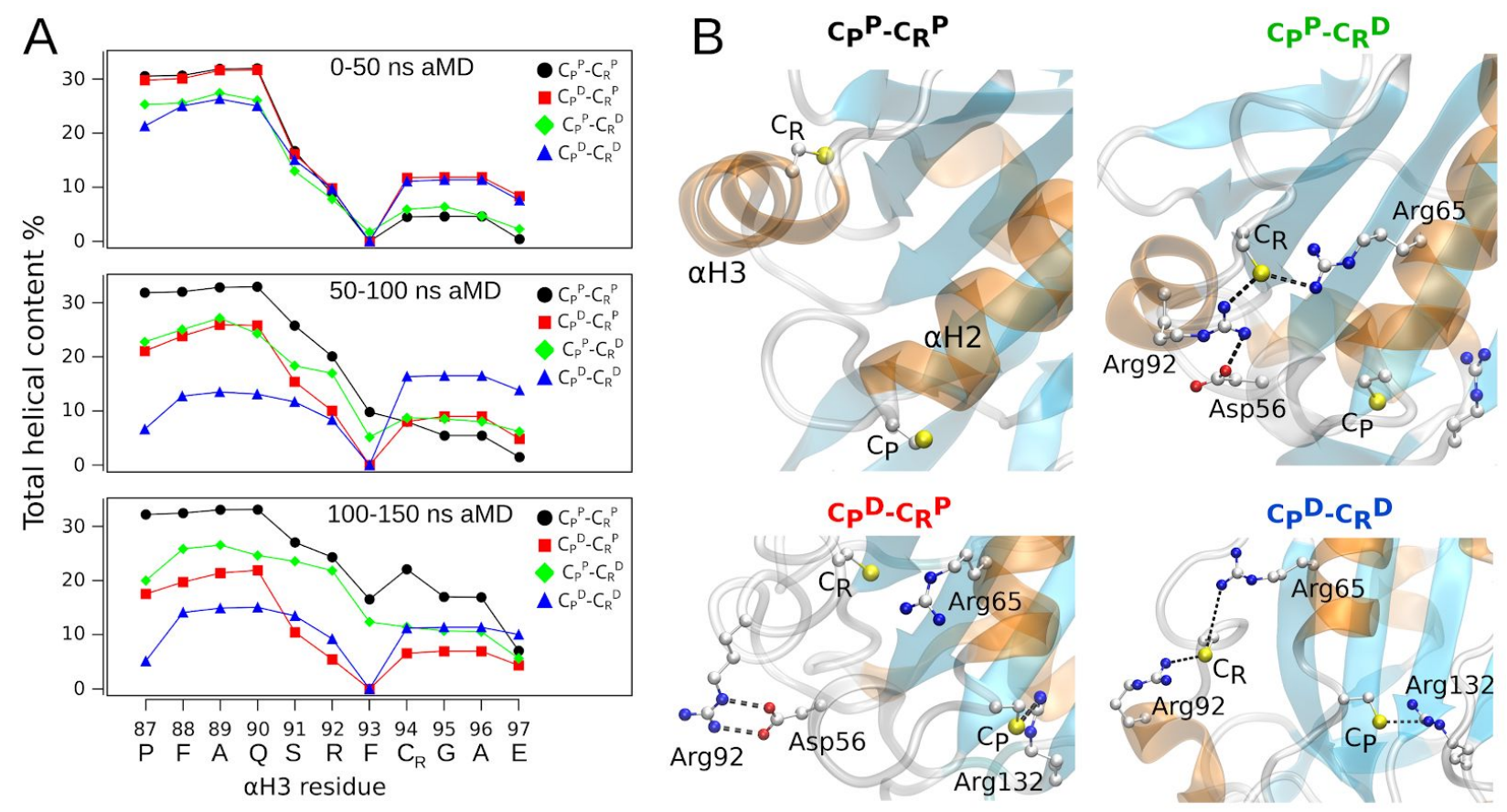

Figure 7: Total Helical Content of the $\alpha H 3$. The percentage helical content of $\alpha \mathrm{H} 3$ for the $\mathrm{C}_{\mathrm{P}}{ }^{\mathrm{P}}-\mathrm{C}_{\mathrm{R}}{ }^{\mathrm{P}}$ (black circles), $\mathrm{C}_{\mathrm{P}}{ }^{\mathrm{P}}-\mathrm{C}_{\mathrm{R}}{ }^{\mathrm{D}}$ (red squares), $\mathrm{C}_{\mathrm{P}}{ }^{\mathrm{D}}-\mathrm{C}_{\mathrm{R}}{ }^{\mathrm{P}}$ (green diamonds) and $\mathrm{C}_{\mathrm{P}}{ }^{\mathrm{D}}-\mathrm{C}_{\mathrm{R}}{ }^{\mathrm{D}}$ (blue up triangles) systems analyzed between 0-50, 50-100 and 100-150 ns of each aMD simulation are shown in panel A. The primary sequence of $\alpha \mathrm{H} 3$ is shown in the $x$-axis. Representative snapshots of each system are shown in panel B. The superscript labels $\mathrm{P}$ and $\mathrm{D}$ correspond to the protonated or deprotonated state of the catalytic Cys, respectively.

\section{Effects of $\alpha \mathrm{H} 3$ Helix Single-point Mutations on the FF Conformational Ensemble}

The analysis of the interactions in FF or LU ensembles showed that the FF and LU conformations are stabilized by different hydrogen bond (HB) networks. The segment comprised between residues 86 to 92 , involving the loop connecting the $\beta 6$ and the first half of $\alpha \mathrm{H} 3$, is strongly stabilized by HBs in the FF ensemble and hydrophobic interactions of Phe93 side chain 
with the protein core (Figure 8, left panel). Noticeably, the second half of $\alpha \mathrm{H} 3$ helix (residues 93 to 97 ), containing the $C_{R}$, is stabilized by only one interaction between Glu97 and Arg65. On the other hand, in the LU ensemble, three HB are formed in the same region: two HB between the side chain $\mathrm{N}^{\zeta}$ of Arg65 with the backbone oxygen of Glu97 and $\mathrm{C}_{\mathrm{P}}$, and one between the backbone oxygen of Phe93 with the backbone N-amide of Glu97; plus the covalent disulfide bond that globally stabilizes the LU conformations (Figure 8, right panel).
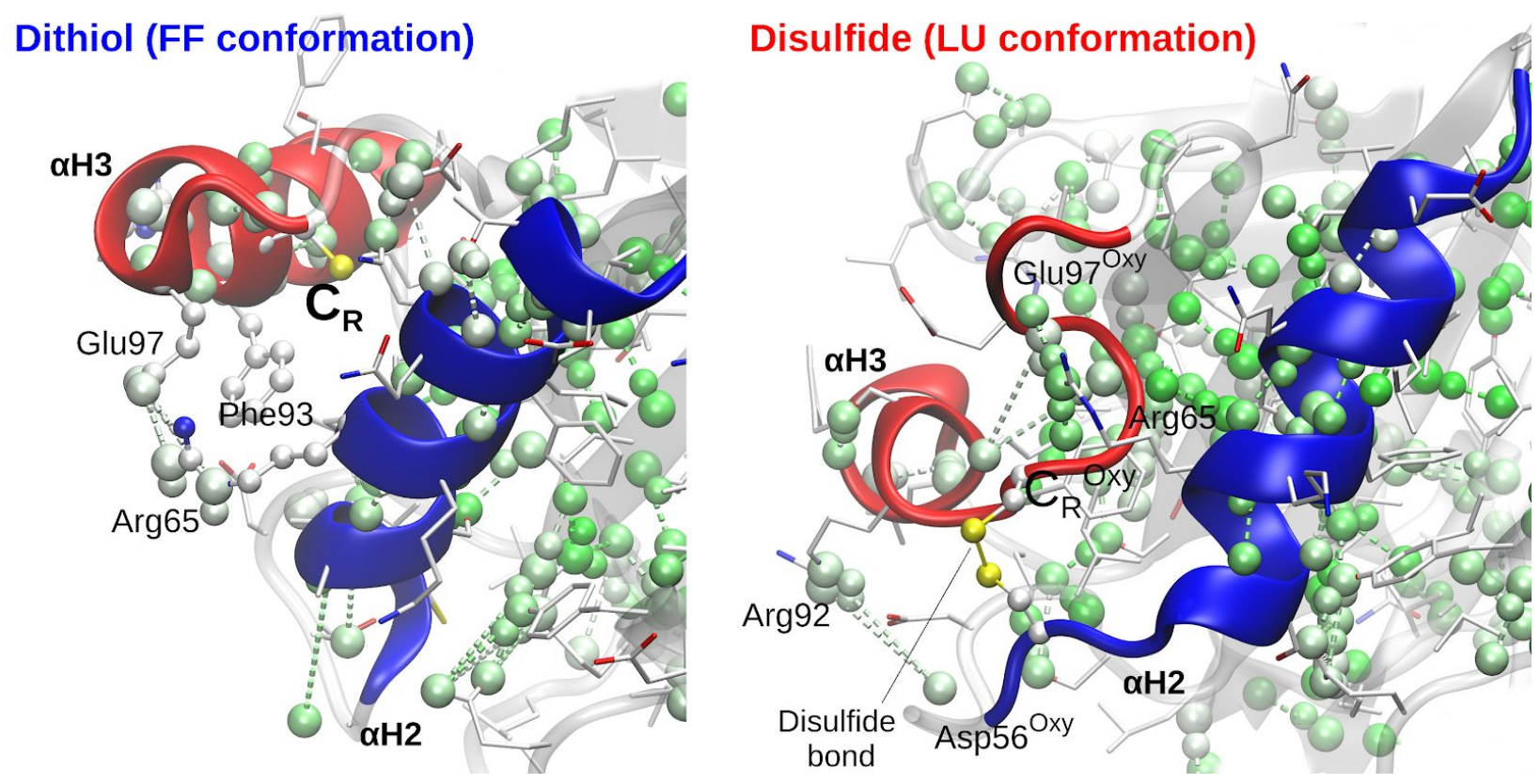

Figure 8: Hydrogen Bond Network Analysis. The HBN was calculated using HBonanza [27] from the cMD trajectories of wild-type EcTPx in the reduced (left panel) and oxidized states (right panel).

To evaluate the effect of altering the HB network and helix propensity, three point mutants were evaluated by MDs, R92G, F93G, and E97G. It is worthy to mention that Gly mutations have two simultaneous effects: first, the truncation of the complete side-chain of the residue and second, the increment of conformational freedom and consequently, the 
destabilization of helical conformations. The three systems were studied throughout $2 \mu \mathrm{s}$ cMD simulations. Variants R92G, F93G, and E97G showed RMSD values of $1.3 \pm 0.2 \AA, 1.6 \pm 0.2 \AA$ and $1.9 \pm 0.3 \AA$, respectively. However, significant changes occurred, as inferred by the SSC analysis of $\alpha \mathrm{H} 3$ (Figure 9). As expected, wild-type EcTPx and R92G mutation are not perturbed along the simulation either in the SSC of $\alpha \mathrm{H} 3$ nor the $\mathrm{C}_{\mathrm{P}}-\mathrm{C}_{\mathrm{R}}$ distance which kept at $13.9 \pm 2.0 \AA$ in concordance to the wild-type variant (average distance $C_{P}-C_{R} 12.3 \pm 2.7 \AA$, Figure 9A and B). On the contrary, both F93G and E97G mutants show several unfolding transition events of the $\alpha \mathrm{H} 3$ helix, concomitantly with the approach of the catalytic Cys to a disulfide bond compatible distance (Figure 9C and D). 

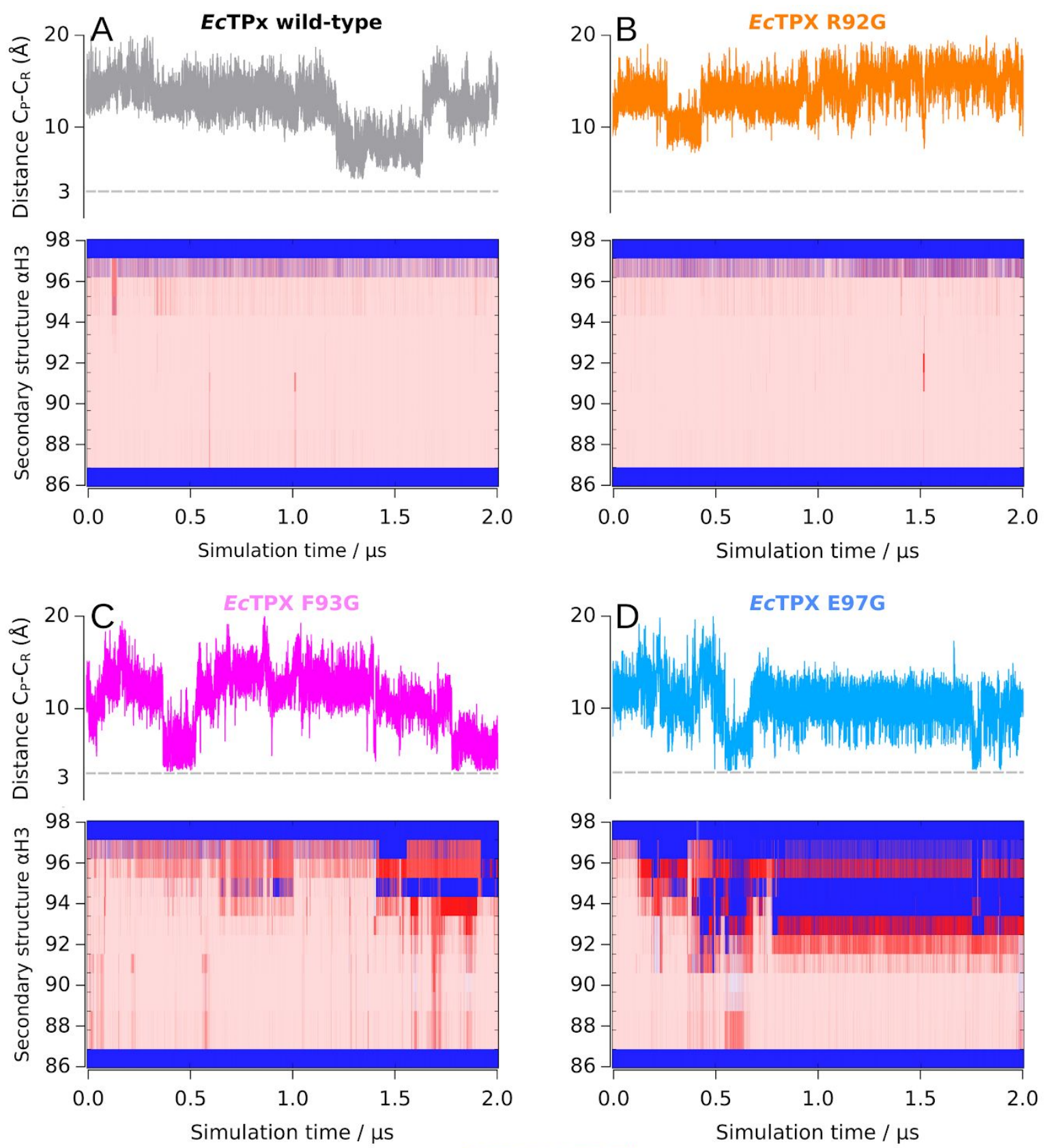

Figure 9: Structural Analysis of EcTPx $\alpha H 3$ Mutants. The $\mathrm{C}_{\mathrm{P}}-\mathrm{C}_{\mathrm{R}}$ distance together with the SSC calculated by the DSSP algorithm [28] of the $\alpha \mathrm{H} 3$ helix are shown for the wild-type variant (A) 
and the point mutants R92G (B), F93G (C) and E97G (D). The grey dotted line indicates a distance of $3 \AA$. ND: non-determined.

\section{Discussion}

The study of connections among protein structure, conformational stability, protein dynamics and function is currently an area of intense efforts. The relationships among these key-features define the energy-functional landscape of proteins and comprehend how it occurs is critical to understand enzyme catalysis. Proteins and pathways that include enzymes belonging to thioredoxin (TRX) superfamily have been well established as system models for this kind of studies. Members with very different structural features, mechanisms of action and activities have been studied. In this context, we decided to study experimentally and computationally the enzyme EcTPx, a protein that shares the TRX fold and emerges as an interesting case in the Prx subfamily.

The protein was successfully overexpressed in E. coli and purified to homogeneity, and further analyzed under reduced and oxidized conditions in experimental and computational studies. Although the enzyme was previously described as a dimer, as suggested by ultracentrifugation experiments at high concentrations (above $40 \mu \mathrm{M}$, [8]), our results demonstrate that, close to physiological conditions and in both redox states, it behaves as a monomer (Figure 2D). The oligomeric features and its influence on the different functions of Prxs is a matter of debate $[3,29,30]$. Most Prxs behave as homo-oligomers (dimers, decamers, etc.) and changes in the quaternary structure are very dependent on a variety of experimental or cellular conditions [31,32]. Nevertheless, a few reports on members of the TPx and PrxQ subfamilies have already shown by applying different techniques that some Prxs behave as 
monomers, such as the case of BsTPx [19], PrxQ from Arabidopsis thaliana [33] and PrxQ B from Mycobacterium tuberculosis [34]. Although key secondary structure rearrangements linked to protein function are present when comparing different redox states of EcTPx, no significant differences in unfolding free energies were observed under reducing and oxidizing conditions. Remarkably, this behavior contrasts with that observed for EcTRX enzyme, the oxidoreductase partner of EcTPx in the catalytic cycle (see Figure 1B). When both redox states of the EcTRX are compared no conformational change are present and only subtle changes in internal motions are observed [16,35,36], whereas the difference in the free energy of unfolding for oxidized state

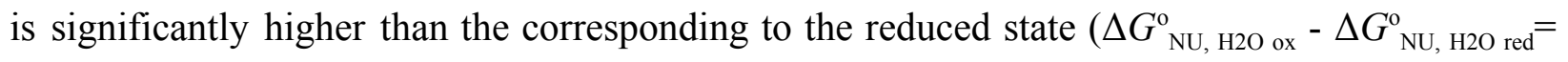
$3.5 \mathrm{kcal} \mathrm{mol}^{-1}$ at $\left.25{ }^{\circ} \mathrm{C},[37,38]\right)$. More experiments should be done to understand why such different behaviors were selected through evolution and to elucidate if there is a thermodynamic connection between both proteins.

Structural evidence provided by X-ray crystallography concerning different states of AhpC from Salmonella typhimurium initially allowed to infer the existence of complex relationships between conformational exchange and activity, pointing to the existence of at least two different and functionally relevant native substates [20]. In the case of the TPx subfamily, the catalytic reaction comprises a partial local unfolding/refolding event of two non-consecutive alpha-helical elements, bringing the intermediate $\mathrm{C}_{\mathrm{P}}-\mathrm{SOH}$ and $\mathrm{C}_{\mathrm{R}}$ species close enough to react, yielding a disulfide bond and releasing a water molecule (see Figure 1B). This suggests that conformational exchange and catalysis are coupled. In particular, the crystallographic structures of reduced and oxidized states of EcTPx have shed light on the conformation of the native substates FF and LU. However, no sufficient in-depth dynamic and mechanistic information 
concerning the conformational transition and protein motions involved is available at the molecular level.

We investigated the $\mathrm{FF} \rightarrow \mathrm{LU}$ and $\mathrm{LU} \rightarrow \mathrm{FF}$ conformational transitions of EcTPx by computational methods. Conventional MDs allowed us to explore different substates of the conformational landscape. In addition, the different oxidation and protonation states of the catalytic Cys residues involved in the reaction were evaluated. This approach was insufficient to investigate the conformational transition. However, the implementation of aMD allowed an enhancing of conformational sampling. Using this technique, we observed the LU $\rightarrow$ FF refolding transition suggesting that the process involves high energetic barriers.

It will be very important to study the molecular nature of the barrier, and the degree of coupling between the internal motions, conformational stability of the helical secondary structure elements and the speed of the resolution steps (conformational transition and disulfide bond formation). Our computational results allowed us the study of the LU $\rightarrow$ FF refolding process, but the $\mathrm{FF} \rightarrow \mathrm{LU}$ transition was not observed as such. This conclusion is in agreement with experimental results regarding the secondary structure content (circular dichroism), that indicated that in equilibrium under reducing conditions, an increase in the helical content is observed, which is compatible with the stabilization of the FF ensemble, relative to the LU ensemble, in aqueous solution. Similar behaviors were previously observed for the atypical 2-Cys TPx from H. pylori [20] and 2-Cys PrxQ B from M. tuberculosis [34].

One of the main questions concerning Prx activity is whether the oxidation/protonation state of the $\mathrm{C}_{\mathrm{P}}$ might trigger the conformational transition or, on the other hand, there is an inherent equilibrium between FF and LU conformations independent of the oxidation and/or 
protonation state of $\mathrm{C}_{\mathrm{P}}$ and $\mathrm{C}_{\mathrm{R}}$. The available data in the literature is controversial. Some results support the idea that $\mathrm{LU}$ conformations can be reached only upon $\mathrm{C}_{\mathrm{P}}$ oxidation to sulfenic acid [39], while others suggest that the FF/LU equilibrium is independent of the oxidation state of $\mathrm{C}_{\mathrm{P}}$ $[33,40]$, but in any case, it could be very dependent on each particular Prx. To shed light on this debatable issue, starting from FF and LU conformations, we modeled $\mathrm{C}_{\mathrm{P}}$ in the thiol, thiolate, sulfenic acid and sulfenate species and subjected to microsecond long cMD simulations. Our MD results suggest that the thiolate species of $\mathrm{C}_{\mathrm{P}}$ stabilize the $\mathrm{FF}$ conformations. This idea is in agreement with the fact that the FF conformations are capable to bind and react with the peroxide substrate [41] since the $\mathrm{pKa}$ value of $\mathrm{C}_{\mathrm{P}}$ is typically below 7 [42,43]. However, when aMDs were carried out from the LU conformations we observed that the thiolate form of the Cys residues may establish ionic par interactions that stabilize the LU conformations. On the other hand, when Cys residues were protonated the LU to FF transition occurred. It is interestingly to note that when the disulfide bond of a Prx (in the LU conformations) is reduced by an oxidoreductase (e.g. TRX), consecutive thiol/disulfide exchange reactions release $C_{P}$ and the $C_{R}$ both transiently as thiolates [44-46] and then they could receive a proton from the solvent or a neighboring acidic residue. Remarkably, $C_{P}$ and $C_{R}$ as thiols is the protonation state that favors the return to the FF conformations in our simulations. Therefore, we inferred that the deprotonation of the Cys residues can modulate the energetics of the conformational exchange altering the barrier between both conformational ensembles. Protonated states might favour the LU $\rightarrow$ FF transition, whereas deprotonation might stabilize the existing conformation. A major caveat needs to be given at this point, the reduction reaction and potentially the $\mathrm{LU} \rightarrow \mathrm{FF}$ transition happens with the TRX bound to the Prx, such binding (transiently covalent) will importantly alter the electrostatic and 
solvation environment surrounding the TPx disulfide. TRX is negatively charged at neutral $\mathrm{pH}$ $(\mathrm{pI}=4.5)$, the interactions between TPx and TRX are mostly electrostatic and may play a central role in the refolding process.

The conformational exchange between two substates of the native ensemble that allows the formation of the disulfide bond in the active site occurs in other Prxs, including the decameric/dimeric Prx1 and Prx2 in which the reduction of the intersubunit disulfide bonds results in an increase in the secondary structure content $[47,48]$. An analogue behaviour was previously described in detail for PrxQ, an atypical 2-Cys Prx, which is exclusively found in bacteria, fungi and plants. However, in PrxQ, catalytic Cys is located prototypically in $\alpha \mathrm{H} 2$ and typically separated by four residues $\left(\mathrm{C}_{\mathrm{P}} \mathrm{xxxxC_{R }}\right)$, although they can also be functional as 1-Cys [49]. Most PrxQ are monomeric and undergo a significant conformational change upon reduction of the disulfide bond [34]. In the case of $A t \operatorname{PrxQ}$, thermal unfolding analysis showed that the oxidized enzyme exhibited lower conformational stability than that the reduced enzyme (48 and $53{ }^{\circ} \mathrm{C}$, respectively, [33]). For the oxidized state, it was observed a significant increase in the effective NMR- $\mathrm{R}_{2}$ relaxation rates in AtPrxQ for residues located in the vicinity of the active site [33], compatible with a conformational exchange and localized slow motions in this region of the protein, confirmed by the measurement of $R_{\text {ex }}$ in CPMG experiments (Carr-Purcell-Meiboom-Gill pulse). This behavior was not observed under reduced conditions [33].

The resolution step (Reaction II, Figure 1), that includes the FF $\rightarrow$ LU transition, has been shown to be significantly slower than expected for a reaction between a sulfenic acid and a thiol. The rate of resolution is differentially regulated in different Prx and sometimes imposes a severe 
limitation in the turnover number of Prx [23]. Additionally, changes in the protein sequence can accelerate the resolution step, this has been shown in the cases of Salmonella typhimurium AhpC W32F (twofold acceleration, [50]), a mycothiol peroxidase from Corynebacterium glutamicum (fivefold acceleration, [51]) and human Prx5 with 22 additional amino acids at the C-terminal (eightfold acceleration, Semelak et al. 2019, unpublished results).

Thus, it seems that the conformational exchange between FF and LU substates of the native state may become a rate limiting and is a key step in the catalytic mechanism of Prx regardless the location of the Cys residues: (i) same secondary structure element, (ii) different secondary structure element but located in the same protein subunit, or (iii) Cys residues located in different subunits. It is expected that different Prxs subfamilies exhibit particularities in the FF/LU equilibrium, depending on the protein primary sequence and topology features. Whether or not the molecular characteristics of this step can define important aspects of kinetics is an important question that should be further studied in detail and in a case-by-case basis.

In addition, mutations that destabilize the FF interaction network (particularly F93G and E97G) resulted, not only in increasing probabilities of local unfolding events of the $\alpha \mathrm{H} 3$, but also in the modulation of the distance that separates the catalytic Cys residues during a significant portion of the MD simulations, which are critical to understand the catalytic features of the enzyme. 


\section{Materials and methods}

\section{Chemicals}

All chemicals reagents were of the purest analytical grade available. Tris(hydroxymethyl)aminomethane (Tris) was from Merck (Darmstadt, HE, DE). Sodium chloride $(\mathrm{NaCl})$ and ethylenediaminetetraacetic acid (EDTA) were from Biopack (Buenos Aires, AR). Isopropyl- $\beta$-d-thiogalactopyranoside (IPTG) was from Bio Basic (Amherst, NY, US). Urea was from ICN Biomedicals Inc. (Irvine, CA, US). Dithiothreitol (DTT) and hydrogen peroxide $\left(\mathrm{H}_{2} \mathrm{O}_{2}\right)$ were from Sigma-Aldrich (St. Louis, MO, US). 4,4'-dithiodipyridine (DTDPy) was from Acros Organics (Hampton, NH, US).

\section{Expression and Purification of $E$. coli Thiol Peroxidase}

The expression vector pPROK1/tpx containing the wild-type E. coli thiol peroxidase (EcTPx) sequence was a gift of Dr. Derek Parsonage (Wake Forest School of Medicine, Winston-Salem, NC, US). The plasmid was transformed to electrocompetent E. coli BL21(DE3) cells and selected using ampicillin $50 \mu \mathrm{g} \mathrm{mL}-1$. The protein was produced in LB medium at $37^{\circ} \mathrm{C}$ to $\mathrm{OD}_{600 \mathrm{~nm}}=0.9$ and overexpression was induced by $0.5 \mathrm{mM}$ IPTG for $4 \mathrm{~h}$ at $37^{\circ} \mathrm{C}$ shaking at $200 \mathrm{rpm}$. Cells were harvested by centrifugation at $5000 \mathrm{~g}$ at $4{ }^{\circ} \mathrm{C}$. Pellets were resuspended in $25 \mathrm{mM}$ Tris-HCl, $1 \mathrm{mM}$ EDTA, pH 7.4 (resuspension buffer) on an ice/water bath. Cells were disrupted by pulse-sonication. Cellular debris and insoluble fraction were removed by centrifugation at $7000 \mathrm{~g}$ at $4{ }^{\circ} \mathrm{C}$. The supernatant fraction containing soluble proteins was loaded onto a sepharose DE52 (Pharmacia Biotech, Uppsala, SE) column equilibrated with $25 \mathrm{mM}$ 
Tris-HCl, $1 \mathrm{mM}$ EDTA, $\mathrm{pH} 7.4$ at $4{ }^{\circ} \mathrm{C}$ and washed with the same buffer. Elution was performed with an increasing gradient up to $1.0 \mathrm{M} \mathrm{NaCl}$ in resuspension buffer. Fractions were evaluated by standard SDS-PAGE procedure [52] and UV absorption spectra and those containing EcTPx were pooled and treated with DNase/RNase $\left(50 \mu \mathrm{gL}^{-1}\right.$ plus $100 \mathrm{mM} \mathrm{Cl} \mathrm{Mg}$ during 12 hours at $4{ }^{\circ} \mathrm{C}$ ), extensively dialyzed against resuspension buffer and then loaded onto a preparative Sephadex G100 (Pharmacia Biotech, Uppsala, SE) size exclusion chromatography (SEC) column $(93 \times 2.7 \mathrm{~cm})$, previously equilibrated with buffer $10 \mathrm{mM}$ Tris- $\mathrm{HCl}, 1 \mathrm{mM}$ EDTA, $100 \mathrm{mM}$ $\mathrm{NaCl}, \mathrm{pH}$ 7.4. Fractions containing EcTPx ( $>95 \%$ pure) were pooled and aliquoted for storage at $-20^{\circ} \mathrm{C}$ until use.

\section{Circular Dichroism Spectroscopy Characterization}

Changes in secondary and tertiary structure content of wild-type EcTPx were followed by the circular dichroism (CD) signal in the far-UV (195 to $260 \mathrm{~nm}$ ) using a $1.0 \mathrm{~mm}$ path-length cell and in the near-UV (240 to $350 \mathrm{~nm}$ ) using a $5 \mathrm{~mm}$ path-length cell. Measurements were made in a Jasco 810 spectropolarimeter (Jasco Corp., Tokio, JP). EcTPx (8-30 $\mu \mathrm{M}$ final concentration) were prepared in $50 \mathrm{mM}$ Tris- $\mathrm{HCl}, 1 \mathrm{mM}$ EDTA $\mathrm{pH}$ 7.4. Both reduced and oxidized states were obtained incubating the protein samples for 30 minutes at room temperature in the presence of $1.0 \mathrm{mM}$ DTT or $1.0 \mathrm{mM} \mathrm{H} \mathrm{O}_{2}$, respectively. After that, DTT or $\mathrm{H}_{2} \mathrm{O}_{2}$ was removed by SEC using a Sephadex G25 matrix (Sigma-Aldrich, St. Louis, MO, US), and the $[\mathrm{SH}] /[$ protein] ratios were checked using DTDPy reagent $[53]\left(\varepsilon_{324 \mathrm{~nm}}=21400 \mathrm{M}^{-1} \mathrm{~cm}^{-1}\right)$. At least ten spectra were acquired at $25 \pm 0.1{ }^{\circ} \mathrm{C}$ using a Peltier system and a speed scan of $50 \mathrm{~nm} \mathrm{~min}{ }^{-1}$ with a time constant of 0.5 s. The proper blanks spectra were acquired, subtracted to the average protein spectrum, smoothed 
using a fourth-degree Savitzky-Golay polynomial filter [54] with a 10-point sliding window and expressed to molar ellipticity using the Equation 1:

$$
[\theta]=\frac{m d e g}{10 \cdot[C] \cdot b \cdot L}
$$

where mdeg is the raw signal in millidegrees, $[\mathrm{C}]$ is the protein concentration in molar units, $b$ is the number of peptide bonds, and $L$ is the path length in centimeters.

\section{Steady-state Chemical-induced Unfolding Followed by Circular Dichroism Spectroscopy}

Equilibrium unfolding experiments were carried out incubating the reduced and oxidized state of EcTPx $(25 \mu \mathrm{M})$ with increased concentrations of urea up to $7.0 \mathrm{M}$ in $50 \mathrm{mM}$ Tris-HCl, 1 mM EDTA, $100 \mathrm{mM} \mathrm{NaCl}, \mathrm{pH} 7.4$ and incubated at room temperature for 5 hours. In the case of the reduced EcTPx, $0.5 \mathrm{mM}$ DTT was added to the buffer to keep reduced conditions. The unfolding process was followed by far-UV CD spectroscopy at $222 \mathrm{~nm}$ using a path length cuvette of $0.1 \mathrm{~cm}$ as a function of time during at least 200 seconds. All measurements were carried out at $25 \pm 0.1^{\circ} \mathrm{C}$. In addition, the far-UV CD spectra at intervals of $1.0 \mathrm{M}$ denaturant were recorder to ensure the loss of structure signatures. A two-state unfolding mechanism (native $[\mathrm{N}] \leftrightarrow$ unfolded $[\mathrm{U}])$ was fitted to the experimental data to calculate thermodynamic parameters $[55,56]$. The reversibility of the process was checked by dilution in both the reduced and oxidized conditions (data not shown). The final concentration of denaturant was determined by refractive index measurements [57] using a Palm Abbe digital refractometer (Misco). 


\section{Oligomeric State in Solution}

Size-exclusion chromatography (SEC) was performed in a FPLC system equipped with an UV absorption detector (Jasco Corp., Tokio, JP) and MALLS detector. SEC experiments were carried out using a Superose 12 (GE Healthcare, Chicago, IL, US) column equilibrated in $50 \mathrm{mM}$ Tris- $\mathrm{HCl}, 100 \mathrm{mM} \mathrm{NaCl} \mathrm{pH} 7.4$ prepared in milliQ water and filtered through a $0.22 \mu \mathrm{m}$ pore size filter. The flow rate was set to $0.3 \mathrm{~mL} \mathrm{~min}^{-1}$ and the injection volume was $100 \mu \mathrm{L}$. Protein samples were prepared at $10 \mathrm{x}\left(1 \mathrm{mg} \mathrm{mL}^{-1}\right)$ to the desired concentration in equilibrium buffer and centrifuged at top speed before injection. In all cases, three independent experiments were performed at room temperature. Data processing was performed using the ASTRA 6.0 software (Wyatt Technology, [58]).

\section{Computational Characterization of the FF and LU Conformational States}

\section{Conventional Molecular Dynamics Protocol}

The atomic coordinates of the reduced (fully folded, FF conformation) and disulfide bonded (locally unfolded, LU conformation) wild-type EcTPx were retrieved from the RCSB Protein Data Bank under the accession codes $3 \mathrm{HVV}$ and $3 \mathrm{HVS}$ at $1.75 \AA$ and $1.80 \AA$ of resolution, respectively [13]. Both crystallographic structures were renumbered throughout this work starting from serine 1 in both cases.

The protonation and oxidation state of the peroxidatic Cys $\left(C_{P} 60\right)$ was changed to model different species of interest (see Table 1). The thiol and thiolate protonation state of $\mathrm{C}_{\mathrm{P}}$ were modeled by the parameters provided in the AMBER package [59]. The parameters for the 
sulfenic acid species were taken from Defelipe et al. [60]. The parameters for the sulfenate species were kindly provided by Dr. Jenner Bonanata (Universidad de la República, Montevideo, Uruguay). The protonation state of the rest of the ionizable residues was set to the corresponding state at neutral $\mathrm{pH}$. The initial coordinates were solvated with the 3-particle TIP3P water model [61] in a cubic box, which extended 10-15 $\AA$ further of the nearest protein atom. The set of parameters for alkali halide ions created by Joung and Cheatham $[62,63]$ were used to balance the total charge of the simulation box. A standard minimization protocol was applied to the resulting structures to remove any clashes for 1000 steps of steepest descent followed by 1000 steps of conjugate gradient minimization procedure in the canonical ensemble (NVT) using periodic boundary conditions. The systems were heating up from $100 \mathrm{~K}$ to $300 \mathrm{~K}$, using the Berendsen thermostat [61] to suppress fluctuations of the kinetic energy of the system with a relaxation constant of 2 ps and subsequently switched to constant isotropic-isobaric (NpT) conditions to allow the density to equilibrate around $\sim 1 \mathrm{~g} \mathrm{~cm}^{-3}$ using the Montecarlo barostat. The SHAKE algorithm [64] was applied to all bonds involving hydrogen atoms, then 2 fs time step was settled. An $8 \AA$ cutoff radius for range-limited interactions with the particle mesh Ewald summation [65] for long-range electrostatic interactions was used. Harmonic positional restraint of strength $20 \mathrm{kcal} \mathrm{mol}^{-1} \AA^{-2}$ on $\mathrm{C} \alpha$ atoms was applied during minimization and equilibration and subsequently removed in four successive simulation stages $\left(10,5,2\right.$ and $\left.0 \mathrm{kcal} \mathrm{mol}^{-1} \AA^{-2}\right)$ of $5 \mathrm{~ns}$ each. Unrestrained cMD simulations were performed with the Amber14 [59] suite using the pmemd.CUDA engine for the graphics processing unit (GPU) code $[66,67]$ in a GeForce GTX780 (Nvidia) equipped cluster and the $f f 14 S B$ force field [68]. Conventional MD runs were 
extended for at least $100 \mathrm{~ns}$ to obtain the parameters needed and the last restart file was used as a starting point to the accelerated MD simulations.

Table 1. Resume of cMD and aMD Simulations Conducted along this Work

\begin{tabular}{|c|c|c|c|c|c|c|c|}
\hline \multirow{2}{*}{$\begin{array}{c}E c \mathrm{TPx} \\
\text { conformational } \\
\text { substate }\end{array}$} & \multicolumn{2}{|c|}{$\begin{array}{c}\text { Protonation and oxidation } \\
\text { states of Cys residues }\end{array}$} & \multirow{2}{*}{$\begin{array}{c}\mathrm{N}_{\text {atoms }} \\
\text { protein }\end{array}$} & \multirow{2}{*}{$\begin{array}{c}\mathrm{N}_{\text {atoms }} \\
\text { system }\end{array}$} & \multirow{2}{*}{$\mathrm{cMD}$} & \multicolumn{2}{|c|}{ aMD } \\
\hline & $\mathrm{C}_{\mathrm{P}}$ & $\mathrm{C}_{\mathrm{R}}$ & & & & $\lambda$ factor & $\begin{array}{c}\text { Simulation } \\
\text { steps }\end{array}$ \\
\hline \multirow{4}{*}{$\mathrm{FF}$} & Thiol & \multirow{4}{*}{ Thiol } & 2502 & 29156 & \multirow{4}{*}{$500 \mathrm{~ns}$} & \multirow{4}{*}{ - } & \multirow{4}{*}{ - } \\
\hline & Thiolate & & 2501 & 29147 & & & \\
\hline & Sulfenic acid & & 2503 & 29157 & & & \\
\hline & Sulfenate & & 2502 & 29148 & & & \\
\hline \multirow{4}{*}{ LU* } & Thiol & \multirow{4}{*}{ Thiol } & 2502 & 27485 & \multirow{4}{*}{$500 \mathrm{~ns}$} & \multirow{4}{*}{ - } & \multirow{4}{*}{ - } \\
\hline & Thiolate & & 2501 & 27473 & & & \\
\hline & Sulfenic acid & & 2503 & 27486 & & & \\
\hline & Sulfenate & & 2502 & 27474 & & & \\
\hline LU & \multicolumn{2}{|c|}{ Disulfide bonded } & 2500 & 29188 & $200 \mathrm{~ns}$ & - & - \\
\hline FF wild-type & \multirow{4}{*}{ Sulfenic acid } & \multirow{4}{*}{ Thiol } & 2503 & 27482 & \multirow{4}{*}{$\begin{array}{c}2000 \\
\text { ns }\end{array}$} & \multirow{4}{*}{ - } & \multirow{4}{*}{ - } \\
\hline FF R92G & & & 2486 & 27473 & & & \\
\hline FF F93G & & & 2490 & 27482 & & & \\
\hline FF E97G & & & 2495 & 27482 & & & \\
\hline \multirow{4}{*}{$\mathrm{FF}$} & Sulfenic acid & Thiol & \multirow{4}{*}{2503} & \multirow{4}{*}{35079} & \multirow{4}{*}{$200 \mathrm{~ns}$} & $\lambda=0.3$ & $10^{8}$ \\
\hline & Sulfenic acid & Thiol & & & & $\lambda=1.0$ & $2.5 \times 10^{8}$ \\
\hline & Sulfenic acid & Thiol & & & & $\lambda=2.0$ & $4 \times 10^{8}$ \\
\hline & Sulfenic acid & Thiol & & & & $\lambda=4.0$ & $10^{9}$ \\
\hline \multirow{4}{*}{ LU* } & Thiol & Thiol & 2502 & 27485 & \multirow{4}{*}{$100 \mathrm{~ns}$} & \multirow{4}{*}{$\lambda=1.0$} & \multirow{4}{*}{$\begin{array}{l}4 \text { replicas } \\
\left(7.5 \times 10^{7}\right)\end{array}$} \\
\hline & Thiol & Thiolate & 2501 & 35432 & & & \\
\hline & Thiolate & Thiol & 2501 & 35432 & & & \\
\hline & Thiolate & Thiolate & 2500 & 35432 & & & \\
\hline
\end{tabular}

FF: the fully folded conformational substate. $\mathrm{LU}$, the locally unfolded conformational substate. LU*: the LU conformational substate lacking the disulfide bond between $\mathrm{C}_{\mathrm{P}}$ and $\mathrm{C}_{\mathrm{R}}$. 


\section{Accelerated Molecular Dynamic Simulation Protocol}

Accelerated molecular dynamics (aMD) is a biased method that enhances conformational sampling [69], promoting the search for low frequency events in biomolecules by adding a non-negative boost potential $\Delta \mathrm{V}(r)$ to the true potential energy function $\mathrm{V}(\mathrm{r})$ when this is lower than a pre-defined energy level E $[25,70,71]$. Such that, the potential surfaces are raised near the minima and unchanged near the saddle points giving as a result, a decrease in the global potential energy barriers. The biased potential function $V^{*}(r)$ is as follows (Equation 2-3):

$$
\begin{gathered}
V^{*}(r)=V(r)+\Delta V(r) \\
\Delta V(r)=\frac{\left(E_{P o t}-V(r)\right)^{2}}{\alpha_{P o t}+E_{P o t}-V(r)}+\frac{\left(E_{D}-V_{D}(r)\right)^{2}}{\alpha_{D}+E_{D}-V_{D}(r)}
\end{gathered}
$$

Where $E_{P o t}$ and $E_{D}$ are the average potential and dihedral energies obtained by a cMD simulation, $\mathrm{V}(r)$ and $\mathrm{V}_{D}(r)$ are the normal potential and torsion potential, respectively, and $\alpha_{P o t}$ and $\alpha_{D}$ are the acceleration factors for the potential and dihedral energies, respectively. The dual-boost method was used in all aMD simulations. $E_{P o t}, E_{D}, \alpha_{P o t}$ and $\alpha_{D}$ are defined as follows:

$$
\begin{gathered}
E_{\text {Pot }}=\left\langle E_{\text {Pot }}^{c M D}\right\rangle+\alpha_{\text {Pot }} \text { and } \alpha_{\text {Pot }}=a \cdot N_{\text {atoms (4) }} \\
E_{D}=\left\langle E_{D}^{c M D}\right\rangle+\alpha_{D}(1+\lambda) \text { and } \alpha_{D}=\frac{b \cdot N_{\text {residue }}}{5}
\end{gathered}
$$

The values of $a$ and $b$ used in the present work were 0.2 and $3.5 \mathrm{kcal} \mathrm{mol}^{-1}$ residue $^{-1}$, respectively. $N_{\text {atoms }}$ and $N_{\text {residue }}$ are the total atoms of the system and the number of residues of the protein, 
respectively. In order to modify the dihedral boost potential, the $\lambda$ parameter was adjusted as described in Equation 5.

\section{General Post-processing Analysis}

Time evolution of the protein secondary structure was calculated with the DSSP algorithm [28] of Kabsch and Sander implemented in the cpptraj routine [72] of AmberTools15. Solvent accessible surface area (SASA) was calculated using the vmdICE plug-in [73] for VMD 1.9.2. The hydrogen bond (HB) network of the cMD trajectories was calculated using the Python-based HBonanza script [27] and visualized using the provided $T c l$ script for VMD 1.9.2 [74]. The analysis was performed over 2000 frames (200 ns of cMD simulation) setting a donor-acceptor distance of $3.0 \AA$ and hydrogen-donor-acceptor angle of 30 degrees. In order to obtain the most stable HBs along the trajectory, all HBs that were formed less frequently than 50 $\%$ of the simulation time were discarded. All protein representations were prepared in VMD 1.9.2 [74].

\section{Principal Component Analysis}

Principal component analysis (PCA) is one of the widest techniques used for the study of the conformational space of protein dynamics $[75,76]$. PCA is a quasi-harmonic analysis involving a linear combination to transforms the original dataset of correlated variables into an uncorrelated reduced dimension and the interatomic distance fluctuation covariance matrix $C_{i j}$ is constructed as follows (Equation 6):

$$
C_{i, j}=\left\langle\left(X_{i}-\left\langle X_{i}\right\rangle\right) \cdot\left(X_{j}-\left\langle X_{j}\right\rangle\right)\right\rangle
$$


where $x_{i, j}$ are the Cartesian atomic coordinates of the atom $i, j$ and $\left\langle x_{i}\right\rangle$ and $\left\langle x_{j}\right\rangle$ denote the average coordinates position over the trajectory. After diagonalization of the covariance matrix, a set of eigenvectors and the corresponding eigenvalues are obtained. The orthogonal eigenvectors set describe axes of the maximal variance of the distribution of conformations, and the eigenvalues provide the variance of atomic positional fluctuations contained along each eigenvector. The root-mean-square inner product $[77,78](\mathrm{RMSIP})$ is a measure for the similarity between the $N$ modes of the covariance matrix of two-mode subspaces obtained by PCA and is calculated by Equation 7:

$$
\operatorname{RMSIP}(i, j)=\sqrt{\frac{\sum_{i=1}^{n} \sum_{j=1}^{n}\left(\eta_{i} \nu_{j}\right)^{2}}{n}}
$$

here $\eta_{\mathrm{i}}$ and $v_{\mathrm{j}}$ are the $i^{t h}$ and $j^{\text {th }}$ eigenvectors of each subspace and $n$ is the number of eigenvectors evaluated. The two limit values of RMSIP are 0 and 1 , where 0 means mutually orthogonal eigenvectors whereas the unity means identical subspaces. In general terms a value of 0.7 is considered an excellent correspondence, a score of 0.5 is fair and below is considered no correlation [77]. The first 10 eigenvectors were used along this work.

\section{Acknowledgments}

This work was supported by grants from the Universidad de Buenos Aires UBACyT N²0020130100468BA, the Agencia Nacional de Promoción Científica y Tecnológica (ANPCyT) PICT N0983/2013, The Centro Argentino Brasileño de Biotecnología (CABBIO) 
PICT-CABBIO N³362/2013 and the Consejo Nacional de Investigaciones Científicas y

Técnicas (CONICET) PIP 2010-2015.

\section{Author Contributions}

DSV, AZ, MM, and GFS designed, planned and performed experiments and analyzed data.

DSV, AZ, and WAA designed and performed computational simulations and analyzed data.

DSV and JS designed and planned all the experiments, analyzed data and wrote the paper. All authors have given approval to the final version of the manuscript.

\section{References}

1 Nelson KJ, Knutson ST, Soito L, Klomsiri C, Poole LB \& Fetrow JS (2011) Analysis of the peroxiredoxin family: using active-site structure and sequence information for global classification and residue analysis. Proteins 79, 947-964.

2 Perkins A, Nelson KJ, Parsonage D, Poole LB \& Karplus PA (2015) Peroxiredoxins: guardians against oxidative stress and modulators of peroxide signaling. Trends Biochem. Sci. 40, 435-445.

3 Zeida A, Trujillo M, Ferrer-Sueta G, Denicola A, Estrin DA \& Radi R (2019) Catalysis of peroxide reduction by fast reacting protein thiols. Chem. Rev. 119, 10829-10855.

4 Karplus PA (2015) A primer on peroxiredoxin biochemistry. Free Radic. Biol. Med. 80, 183-190.

5 Karplus PA \& Hall A (2009) Structural survey of the peroxiredoxins, L. Flohé and J. R: Harris Springer, New York, NY.

6 Cha MK, Kim HK \& Kim IH (1996) Mutation and Mutagenesis of thiol peroxidase of Escherichia coli and a new type of thiol peroxidase family. J. Bacteriol. 178, 5610-5614.

7 Zhou Y, Wan XY, Wang HL, Yan ZY, Hou YD \& Jin DY (1997) Bacterial scavengase p20 is structurally and functionally related to peroxiredoxins. Biochem. Biophys. Res. Commun. 233, 848-852.

8 Baker LMS \& Poole LB (2003) Catalytic mechanism of thiol peroxidase from Escherichia coli. Sulfenic acid formation and overoxidation of essential CYS61. J. Biol. Chem. 278, 9203-9211.

9 Choi J, Choi S, Choi J, Cha M-K, Kim I-H \& Shin W (2003) Crystal structure of Escherichia coli thiol peroxidase in the oxidized state: insights into intramolecular disulfide formation 
and substrate binding in atypical 2-Cys peroxiredoxins. J. Biol. Chem. 278, 49478-49486.

10 Wood ZA, Schröder E, Robin Harris J \& Poole LB (2003) Structure, mechanism and regulation of peroxiredoxins. Trends Biochem. Sci. 28, 32-40.

11 Hall A, Nelson K, Poole LB \& Karplus PA (2011) Structure-based insights into the catalytic power and conformational dexterity of peroxiredoxins. Antioxid. Redox Signal. 15, 795-815.

12 Vazquez DS, Delfino JM \& Santos J (2015) Thioredoxin from Escherichia coli as a Role Model of Molecular Recognition, Folding, Dynamics and Function. Protein Pept. Lett. 22, 801-815.

13 Hall A, Sankaran B, Poole LB \& Karplus PA (2009) Structural changes common to catalysis in the Tpx peroxiredoxin subfamily. J. Mol. Biol. 393, 867-881.

14 Perkins A, Poole LB \& Karplus PA (2014) Tuning of peroxiredoxin catalysis for various physiological roles. Biochemistry 53, 7693-7705.

15 Perkins A, Gretes MC, Nelson KJ, Poole LB \& Karplus PA (2012) Mapping the active site helix-to-strand conversion of CxxxxC peroxiredoxin Q enzymes. Biochemistry 51, 7638-7650.

16 Noguera ME, Vazquez DS, Ferrer-Sueta G, Agudelo WA, Howard E, Rasia RM, Manta B, Cousido-Siah A, Mitschler A, Podjarny A \& Santos J (2017) Structural variability of E. coli thioredoxin captured in the crystal structures of single-point mutants. Sci. Rep. 7, 42343.

17 Trujillo M, Alvarez B \& Radi R (2016) One- and two-electron oxidation of thiols: mechanisms, kinetics and biological fates. Free Radic. Res. 50, 150-171.

18 Sarma GN, Nickel C, Rahlfs S, Fischer M, Becker K \& Karplus PA (2005) Crystal structure of a novel Plasmodium falciparum 1-Cys peroxiredoxin. J. Mol. Biol. 346, 1021-1034.

19 Lu J, Yang F, Li Y, Zhang X, Xia B \& Jin C (2008) Reversible conformational switch revealed by the redox structures of Bacillus subtilis thiol peroxidase. Biochem. Biophys. Res. Commun. 373, 414-418.

20 Nguyen HTM, Nam K-H, Saleem Y \& Kim K-S (2010) Characterization of Helicobacter pylori adhesin thiol peroxidase (HP0390) purified from Escherichia coli. J. Biosci. 35, 241-248.

21 Buchko GW, Perkins A, Parsonage D, Poole LB \& Karplus PA (2016) Backbone chemical shift assignments for Xanthomonas campestris peroxiredoxin $\mathrm{Q}$ in the reduced and oxidized states: a dramatic change in backbone dynamics. Biomol. NMR Assign. 10, 57-61.

22 Cuevasanta E, Reyes AM, Zeida A, Mastrogiovanni M, De Armas MI, Radi R, Alvarez B \& Trujillo M (2019) Kinetics of formation and reactivity of the persulfide in the one-cysteine peroxiredoxin from Mycobacterium tuberculosis. J. Biol. Chem. 294, 13593-13605.

23 Portillo-Ledesma S, Randall LM, Parsonage D, Dalla Rizza J, Karplus PA, Poole LB, Denicola A \& Ferrer-Sueta G (2018) Differential Kinetics of Two-Cysteine Peroxiredoxin Disulfide Formation Reveal a Novel Model for Peroxide Sensing. Biochemistry 57, $3416-3424$. 
24 Amadei A, Linssen AB \& Berendsen HJ (1993) Essential dynamics of proteins. Proteins 17, $412-425$.

25 Hamelberg D, Mongan J \& McCammon JA (2004) Accelerated molecular dynamics: a promising and efficient simulation method for biomolecules. J. Chem. Phys. 120, 11919-11929.

26 Miao Y, Nichols SE, Gasper PM, Metzger VT \& McCammon JA (2013) Activation and dynamic network of the M2 muscarinic receptor. Proc Natl Acad Sci USA 110, 10982-10987.

27 Durrant JD \& McCammon JA (2011) HBonanza: a computer algorithm for molecular-dynamics-trajectory hydrogen-bond analysis. J. Mol. Graph. Model. 31, 5-9.

28 Kabsch W \& Sander C (1983) Dictionary of protein secondary structure: pattern recognition of hydrogen-bonded and geometrical features. Biopolymers 22, 2577-2637.

29 Barranco-Medina S, Lázaro J-J \& Dietz K-J (2009) The oligomeric conformation of peroxiredoxins links redox state to function. FEBS Lett. 583, 1809-1816.

30 Nelson KJ, Perkins A, Van Swearingen AED, Hartman S, Brereton AE, Parsonage D, Salsbury FR, Karplus PA \& Poole LB (2018) Experimentally dissecting the origins of peroxiredoxin catalysis. Antioxid. Redox Signal. 28, 521-536.

31 Morais MAB, Giuseppe PO, Souza TACB, Alegria TGP, Oliveira MA, Netto LES \& Murakami MT (2015) How pH modulates the dimer-decamer interconversion of 2-Cys peroxiredoxins from the Prx1 subfamily. J. Biol. Chem. 290, 8582-8590.

32 Tairum CA, Santos MC, Breyer CA, Geyer RR, Nieves CJ, Portillo-Ledesma S, Ferrer-Sueta G, Toledo JC, Toyama MH, Augusto O, Netto LES \& de Oliveira MA (2016) Catalytic Thr or Ser Residue Modulates Structural Switches in 2-Cys Peroxiredoxin by Distinct Mechanisms. Sci. Rep. 6, 33133.

33 Adén J, Wallgren M, Storm P, Weise CF, Christiansen A, Schröder WP, Funk C \& Wolf-Watz M (2011) Extraordinary $\mu$ s-ms backbone dynamics in Arabidopsis thaliana peroxiredoxin Q. Biochim. Biophys. Acta 1814, 1880-1890.

34 Reyes AM, Vazquez DS, Zeida A, Hugo M, Piñeyro MD, De Armas MI, Estrin D, Radi R, Santos J \& Trujillo M (2016) PrxQ B from Mycobacterium tuberculosis is a monomeric, thioredoxin-dependent and highly efficient fatty acid hydroperoxide reductase. Free Radic. Biol. Med. 101, 249-260.

35 Stone MJ, Chandrasekhar K, Holmgren A, Wright PE \& Dyson HJ (1993) Comparison of backbone and tryptophan side-chain dynamics of reduced and oxidized Escherichia coli thioredoxin using 15N NMR relaxation measurements. Biochemistry 32, 426-435.

36 Kemple MD, Yuan P, Nollet KE, Fuchs JA, Silva N \& Prendergast FG (1994) 13C NMR and fluorescence analysis of tryptophan dynamics in wild-type and two single-Trp variants of Escherichia coli thioredoxin. Biophys. J. 66, 2111-2126.

37 Kelley RF, Shalongo W, Jagannadham MV \& Stellwagen E (1987) Equilibrium and kinetic measurements of the conformational transition of reduced thioredoxin. Biochemistry 26, 
1406-1411.

38 Vazquez DS, Sánchez IE, Garrote A, Sica MP \& Santos J (2015) The E. coli thioredoxin folding mechanism: the key role of the C-terminal helix. Biochim. Biophys. Acta 1854, 127-137.

39 Cao Z, Tavender TJ, Roszak AW, Cogdell RJ \& Bulleid NJ (2011) Crystal structure of reduced and of oxidized peroxiredoxin IV enzyme reveals a stable oxidized decamer and a non-disulfide-bonded intermediate in the catalytic cycle. J. Biol. Chem. 286, 42257-42266.

40 Perkins A, Nelson KJ, Williams JR, Parsonage D, Poole LB \& Karplus PA (2013) The sensitive balance between the fully folded and locally unfolded conformations of a model peroxiredoxin. Biochemistry 52, 8708-8721.

41 Nakamura T, Kado Y, Yamaguchi T, Matsumura H, Ishikawa K \& Inoue T (2010) Crystal structure of peroxiredoxin from Aeropyrum pernix K1 complexed with its substrate, hydrogen peroxide. J. Biochem. 147, 109-115.

42 Winterbourn CC \& Metodiewa D (1999) Reactivity of biologically important thiol compounds with superoxide and hydrogen peroxide. Free Radic. Biol. Med. 27, 322-328.

43 Winterbourn CC \& Hampton MB (2008) Thiol chemistry and specificity in redox signaling. Free Radic. Biol. Med. 45, 549-561.

44 Poole LB (2005) Bacterial defenses against oxidants: mechanistic features of cysteine-based peroxidases and their flavoprotein reductases. Arch. Biochem. Biophys. 433, 240-254.

45 Jönsson TJ, Ellis HR \& Poole LB (2007) Cysteine reactivity and thiol-disulfide interchange pathways in $\mathrm{AhpF}$ and $\mathrm{AhpC}$ of the bacterial alkyl hydroperoxide reductase system. Biochemistry 46, 5709-5721.

46 Lian F-M, Yu J, Ma X-X, Yu X-J, Chen Y \& Zhou C-Z (2012) Structural snapshots of yeast alkyl hydroperoxide reductase Ahp1 peroxiredoxin reveal a novel two-cysteine mechanism of electron transfer to eliminate reactive oxygen species. J. Biol. Chem. 287, 17077-17087.

47 Randall L, Manta B, Nelson KJ, Santos J, Poole LB \& Denicola A (2016) Structural changes upon peroxynitrite-mediated nitration of peroxiredoxin 2; nitrated Prx2 resembles its disulfide-oxidized form. Arch. Biochem. Biophys. 590, 101-108.

48 Dalla Rizza J, Randall LM, Santos J, Ferrer-Sueta G \& Denicola A (2019) Differential parameters between cytosolic 2-Cys peroxiredoxins, PRDX1 and PRDX2. Protein Sci. 28, 191-201.

49 Wakita M, Masuda S, Motohashi K, Hisabori T, Ohta H \& Takamiya K (2007) The significance of type II and PrxQ peroxiredoxins for antioxidative stress response in the purple bacterium Rhodobacter sphaeroides. J. Biol. Chem. 282, 27792-27801.

50 Parsonage D, Nelson KJ, Ferrer-Sueta G, Alley S, Karplus PA, Furdui CM \& Poole LB (2015) Dissecting peroxiredoxin catalysis: separating binding, peroxidation, and resolution for a bacterial AhpC. Biochemistry 54, 1567-1575.

51 Pedre B, Van Molle I, Villadangos AF, Wahni K, Vertommen D, Turell L, Erdogan H, Mateos LM \& Messens J (2015) The Corynebacterium glutamicum mycothiol peroxidase is 
a reactive oxygen species-scavenging enzyme that shows promiscuity in thiol redox control. Mol. Microbiol. 96, 1176-1191.

52 Laemmli UK (1970) Cleavage of structural proteins during the assembly of the head of bacteriophage T4. Nature 227, 680-685.

53 Grassetti DR \& Murray JF (1967) Determination of sulfhydryl groups with 2,2'- or 4,4'-dithiodipyridine. Archives of Biochemistry and Biophysics 119, 41-49.

54 Savitzky A \& Golay MJE (1964) Smoothing and differentiation of data by simplified least squares procedures. Anal. Chem. 36, 1627-1639.

55 Bolen DW \& Santoro MM (1988) Unfolding free energy changes determined by the linear extrapolation method. 2. Incorporation of delta $\mathrm{G}$ degrees N-U values in a thermodynamic cycle. Biochemistry 27, 8069-8074.

56 Santoro MM \& Bolen DW (1988) Unfolding free energy changes determined by the linear extrapolation method. 1. Unfolding of phenylmethanesulfonyl alpha-chymotrypsin using different denaturants. Biochemistry 27, 8063-8068.

57 Grimsley GR, Huyghues-Despointes BMP, Pace CN \& Scholtz JM (2006) Preparation of urea and guanidinium chloride stock solutions for measuring denaturant-induced unfolding curves. CSH Protoc. 2006.

58 Oliva A, Llabrés M \& Fariña JB (2009) Estimation of uncertainty in size-exclusion chromatography with a double detection system (light-scattering and refractive index). Talanta 78, 781-789.

59 Case DA \& Others (2015) AMBER 2015. University of California, San Francisco.

60 Defelipe LA, Lanzarotti E, Gauto D, Marti MA \& Turjanski AG (2015) Protein topology determines cysteine oxidation fate: the case of sulfenyl amide formation among protein families. PLoS Comput. Biol. 11, e1004051.

61 Jorgensen WL, Chandrasekhar J \& Madura JD (1983) Comparison of simple potential functions for simulating liquid water. The Journal of Chemical Physics 79.

62 Joung IS \& Cheatham TE (2009) Molecular dynamics simulations of the dynamic and energetic properties of alkali and halide ions using water-model-specific ion parameters. $J$. Phys. Chem. B 113, 13279-13290.

63 Joung IS \& Cheatham TE (2008) Determination of alkali and halide monovalent ion parameters for use in explicitly solvated biomolecular simulations. J. Phys. Chem. B 112, 9020-9041.

64 Ryckaert J-P, Ciccotti G \& Berendsen HJ. (1977) Numerical integration of the cartesian equations of motion of a system with constraints: molecular dynamics of n-alkanes. $J$. Comput. Phys. 23, 327-341.

65 Leach A (2001) Molecular Modelling: Principles and Applications, Second Ed. Prentice Hall.

66 Götz AW, Williamson MJ, Xu D, Poole D, Le Grand S \& Walker RC (2012) Routine Microsecond Molecular Dynamics Simulations with AMBER on GPUs. 1. Generalized Born. J. Chem. Theory Comput. 8, 1542-1555. 
67 Salomon-Ferrer R, Götz AW, Poole D, Le Grand S \& Walker RC (2013) Routine Microsecond Molecular Dynamics Simulations with AMBER on GPUs. 2. Explicit Solvent Particle Mesh Ewald. J. Chem. Theory Comput. 9, 3878-3888.

68 Maier JA, Martinez C, Kasavajhala K, Wickstrom L, Hauser KE \& Simmerling C (2015) ff14SB: Improving the Accuracy of Protein Side Chain and Backbone Parameters from ff99SB. J. Chem. Theory Comput. 11, 3696-3713.

69 Miao Y, Feixas F, Eun C \& McCammon JA (2015) Accelerated molecular dynamics simulations of protein folding. J. Comput. Chem. 36, 1536-1549.

70 Hamelberg D, de Oliveira CAF \& McCammon JA (2007) Sampling of slow diffusive conformational transitions with accelerated molecular dynamics. J. Chem. Phys. 127, 155102.

71 Markwick PRL \& McCammon JA (2011) Studying functional dynamics in bio-molecules using accelerated molecular dynamics. Phys. Chem. Chem. Phys. 13, 20053-20065.

72 Roe DR \& Cheatham TE (2013) PTRAJ and CPPTRAJ: software for processing and analysis of molecular dynamics trajectory data. J. Chem. Theory Comput. 9, 3084-3095.

73 Knapp B, Lederer N, Omasits U \& Schreiner W (2010) vmdICE: a plug-in for rapid evaluation of molecular dynamics simulations using VMD. J. Comput. Chem. 31, $2868-2873$.

74 Humphrey W, Dalke A \& Schulten K (1996) VMD: visual molecular dynamics. J. Mol. Graph. 14, 33-8, 27.

75 Maisuradze GG, Liwo A \& Scheraga HA (2009) Principal component analysis for protein folding dynamics. J. Mol. Biol. 385, 312-329.

76 Alakent B, Doruker P \& Camurdan MC (2004) Application of time series analysis on molecular dynamics simulations of proteins: a study of different conformational spaces by principal component analysis. J. Chem. Phys. 121, 4759-4769.

77 Amadei A, Ceruso MA \& Di Nola A (1999) On the convergence of the conformational coordinates basis set obtained by the essential dynamics analysis of proteins' molecular dynamics simulations. Proteins 36, 419-424.

78 Leo-Macias A, Lopez-Romero P, Lupyan D, Zerbino D \& Ortiz AR (2005) An analysis of core deformations in protein superfamilies. Biophys. J. 88, 1291-1299. 